\title{
PIECEWISE LINEAR MODELS \\ FOR THE \\ QUASIPERIODIC TRANSITION TO CHAOS.
}

\author{
February 2, 1995
}

David K. Campbell ${ }^{1}$, Roza Galeeva ${ }^{2}$, Charles Tresser ${ }^{3}$, and David J. Uherka ${ }^{4}$

\begin{abstract}
We formulate and study analytically and computationally two families of piecewise linear degree one circle maps. These families offer the rare advantage of being non-trivial but essentially solvable models for the phenomenon of mode-locking and the quasi-periodic transition to chaos. For instance, for these families, we obtain complete solutions to several questions still largely unanswered for families of smooth circle maps. Our main results describe (1) the sets of maps in these families having some prescribed rotation interval; (2) the boundaries between zero and positive topological entropy and between zero length and non-zero length rotation interval; and (3) the structure and bifurcations of the attractors in one of these families. We discuss the interpretation of these maps as loworder spline approximations to the classic "sine-circle" map and examine more generally the implications of our results for the case of smooth circle maps. We also mention a possible connection to recent experiments on models of a driven Josephson junction.

\footnotetext{
1 Physics Department, University of Illinois, 1110 W. Green St., Urbana, IL 61801.

2 UMPA, ENS, 46 Allee d'Italie 69364 Lyon Cedex 07, France

3 I.B.M. Po Box 218, Yorktown Heights, NY 10598.

4 Mathematics Department, University of North Dakota, Grand Forks, ND 58202-8376.
} 


\section{Introduction.}

The phenomenon of "mode locking", in which two (or more) coupled nonlinear oscillators having in general irrationally related ("incommensurate") intrinsic frequencies lock into a periodic motion involving a rational ("commensurate") ratio of their actual frequencies, has been observed and studied experimentally at least since the time of Huyghens $[\mathrm{Hu}]$, who discovered that two pendulum clocks mounted on a common wall tended to "synchronize" their motions, so that both their frequencies and phases became "locked" together. Physical systems ranging from convecting fluids [SHL],[MSE] through nonlinear electrical conductors [MM, BBJ] to chemical reactions [MS] have been shown experimentally to exhibit not only synchronization (1:1 mode locking) but also mode locking into many other rational ratios ("p:q mode locking," for general integers p and q). Further, although one is generally interested in mode-locking in the temporal behavior (i.e., the dynamics) of coupled oscillators, analogous phenomena can occur in the spatial structure of certain solids in which interactions with two different length scales compete [Bak], [BBr], $[\mathrm{BrB}]$.

In the biological realm, mode locking is both widespread and of considerable importance to life processes. Already in the early stages of the modern development of nonlinear dynamics, the human heart was modeled as a nonlinear dynamical system [VdPVdM], and the problem of mode locking in the context of cardiac arrhythmias was studied in one of the central rigorous works on nonlinear dynamics (see [Ar2] for a report of this early work). Since then mode locking has been shown to be nearly ubiquitous in biological systems, occurring for instance (in addition to cardiac arrhythmias [PG], [Gl], [GB]) in the firings of neurons, in animal gaits, and in the coupling of breathing and locomotion (for a concise elementary introduction and references, see [StSt], and for more detailed accounts and references, see $[\mathrm{St}],[\mathrm{GM}],[\mathrm{Wi}])$. It is thus not surprising that attempts to develop a detailed, quantitative understanding of mode locking - and of the associated "quasiperiodic transition to chaos" [FKS],[JBB1],[JBB2], [ORSS] - have played a central role in modern nonlinear dynamics and chaos theory. Many of the applications to physical and biological systems have focused on what is perhaps the simplest model of mode 
locking: continuous mappings of the circle onto itself. First introduced by Poincaré in his efforts to model the motion of trajectories on tori, orientation-preserving homeomorphisms and diffeomorphisms of the circle continue to attract the attention of mathematicians both as interesting and challenging dynamical systems in their own right and in their original context as Poincaré maps induced by non-singular flow on the two-dimensional torus [De], [Ar], [He]. More recently, families of circle endomorphisms which are deformations of rotations have appeared as approximate models for the transition to chaos (as defined, for instance, as the transition from zero to positive topological entropy) in flows in 3-spaces where tori supporting non-singular flows become wrinkled and then destroyed as some parameters are varied (see, e.g., [CTA], [Boyl], [MaT] and references therein).

The classic example of a circle endomorphism is the so-called standard circle or sinecircle map, defined by the two-parameter family :

$$
f_{a, b}: \theta \mapsto\left(\theta+a+\frac{b}{2 \pi} \cdot \sin (2 \pi \theta)\right)_{1}
$$

with $(a, b) \in\left[0,1\left[\times \mathbb{R}^{+}\right.\right.$, and where we have used the notation:

$$
(z)_{n} \stackrel{\text { def }}{=} z \bmod n
$$

The two parameters in $f_{a, b}$ correspond to the non-linearity $(b)$ and the bare mean rotation speed $(a)$, i.e., the mean speed at which the map winds around the circle in the absence of nonlinearity. In the natural science context of coupled oscillators, the two parameters correspond to the strength of the coupling (the forcing of the oscillators on each other) and the ratio of the individual frequencies of the two oscillators, respectively. Note that the mean rotation speed emerges as a property of the map, and one can generally control only the bare mean rotation speed.

More generally, one would like to understand the dynamics of maps, organized for instance in two-parameter families of the form

$$
g_{a, b}: \theta \mapsto\left(\theta+a+h_{b}(\theta)\right)_{1}
$$

where $h_{b}$ is a one parameter family of maps on the unit interval for which 0 and 1 are fixed points (see [GTr] for a review). Whenever $h_{b}$ is a family of smooth maps, the 
description of the family $g_{a, b}$ becomes a very difficult problem, many aspects of which are still beyond our analytic grasp, despite important recent progress (see, e.g., [Me], [EKT] and references therein). Although computational simulations and heuristic calculations can in many cases provide considerable insight, one naturally seeks a more tractable class of models from which to draw analytic guidance. In this regard, it is useful to recall our experience with the extensively analyzed one-parameter endomorphisms of the interval. Here, the paradigm is the quadratic family, exemplified by the familiar "logistic map",

$$
f_{r}: x \mapsto r x(1-x)
$$

Many of the results which are quite difficult in the context of the logistic map can be fairly easily established if one considers the case of piecewise linear maps: the tent map and the trapezoidal map are the two simplest examples, but higher-order piecewise linear maps (such as the "house" map [Ga2], [HC]) have also been studied.

Following these clues, we will focus our present study to the case in which $h_{b}$ is piecewise linear, so that our circle map has what one might call a "sawtooth" form. We stress, of course, that even for piecewise linear circle maps, as in the case of maps on the interval, a number of questions remain deep, difficult, and open. Moreover, in the cases of both interval and circle maps, piecewise linear maps contain "pathologies," in the sense that some aspects of their dynamics and bifurcation structure are different from what happens in the smooth case. Many of these pathologies are intimately related to the (easy) solvability of some questions. Nonetheless, as we shall establish, one can readily gain nearly complete analytic insight into the bifurcation structure of piecewise linear circle maps. Further, this insight is such that many of our results admit rather easy proofs (although not all of them were that easy to find), and methods are most often elementary. Thus a second important feature of these piecewise linear maps is that they provide excellent pedagogical examples allowing non-specialists to gain insight into what physicists would call a non-trivial, solvable model for the quasi-periodic transition to chaos. This has motivated us to try to keep the bulk of the paper accessible to non-specialists (and, in particular, non-mathematicians). That the paper is accessible does not mean that it is easy reading, since for compactness and precision, most of our article is couched in 
mathematical terminology. Hence non-mathematicians will likely wish to refer to Appendix A, which provides the essential definitions and background material, before commencing the body of the article; let us also mention [MSt] as a recent general reference on onedimensional dynamics. A third motivation for our study was the possibility that detailed examination of the piecewise linear models might lead to novel conjectures or suggest methods of proof for broader classes of maps, including the smooth case. Indeed, as we discuss below, this possibility has already been realized, and several new results on the smooth have been stimulated by results obtained in this study. (see, e.g., [EKT],[GMT]).

The present article is the sequel to the announcement made in [UTGC], which was itself in turn motivated by an unpublished manuscript by two of us (D.K.C. and D.J.U.), dealing mainly with invertible maps. In outline, we have organized the remainder of the paper as follows (refer to Appendix A for definitions of the few technical terms used here). In $\S 2$, we describe the two types of piecewise linear maps we consider, the tip maps and the plateau maps ( $c f$ Figure 1). In $\S 3$, we organize these maps in parametrized families. We argue that both the tip maps and the plateau maps are most naturally organized in three-parameter families, which we slice into two-parameter families for the ease of the discussion, to permit clearer graphical illustrations, and for purposes of comparison with the standard circle map. In $\S 4$, we associate to any map $f$ a pair of monotone maps useful in the study of $f$. In $\S 5$, we define subsets of the parameter spaces, subsets whose descriptions amount to capturing some essential aspects of the bifurcation structure. In $\S 6$, we give some general results, counter-parts of known results for smooth families. In $\S 7$, we describe the bifurcation structure for invertible maps and for monotone but non-invertible maps. In $\S 8$, we describe the sets of maps with a given rotation interval. In $\S 9$, we assemble all our main results on the topology and geometry of the boundaries of topological chaos (meaning positive topological entropy) and rotational chaos (meaning the rotation interval has non-zero length). These results rely on some specific properties of tip maps proved in $\S 10$, including aspects of the dynamics of tip maps and their bifurcation structure, primarily involving periodic attractors and related questions. Finally, in $\S 11$, we present some general conclusions, open problems, and a discussion of possible physical realizations of these piecewise-linear maps. In Appendix A, we have assembled some definitions and 
classic background material on subjects including degree, lift, rotation number and rotation interval with their basic properties, Denjoy theory, and topological entropy. Appendix B also contains (some more advanced) background material for the main text. It describes stunted families (see also [BMT], [DGMT] and references therein) and concludes with a new result for such families, which is used in $\S 9$. Appendix $\mathrm{C}$ explains how to compute the boundaries of some of the regions described in $\S 5$. Appendix D describes numerical computations about the likelihood of irrational rotation number for invertible maps. These computations provide support for a conjectured result for which we mistakenly thought we had a proof in [UTGC]: interestingly enough, the numerical evidence was gathered only after we realized our proof was faulty.

Throughout the main text and the appendices, all fractions will be written in lowest order terms, except where otherwise specified. The theorems in the main text carry a number and a name which is an abstract of their content: they are original (to the best of our knowledge), except where otherwise specified. Some theorems are strictly speaking new, but their proofs are merely rewritten versions of similar proofs for smooth maps: this is acknowledged on each occasion . The reason we include these proofs is that the original proofs are not only scattered throughout the literature but are also often published in papers substantially more technical than the present one. The theorems in the appendices carry an uppercase letter or the name of their author: they are (well) known, unless we indicate otherwise. Most technical words appear first (except in this introduction) in italicized form, followed by their definition or endowed with a subscript which refers to a section of an appendix in which the definition is given. Finally, we note that on occasion we will quote later theorems in the proofs of earlier ones; this is done from continuity and clarity in the presentation. The essence of the problem being nonlinear, one could hardly expect a totally linear exposition to be adequate! 


\section{The maps.}

We use the term circle map to denote for short, a continuous map from the circle $\mathbb{I}=\mathbb{R} / \mathbb{Z}$ - we use the standard notation in which $\mathbb{R}$ denotes the set of real numbers and $\mathbb{Z}$ the integers- to itself which has degree one $e_{A-1}$. In this paper, we consider two specific classes of piecewise-linear, degree-one circle maps, whose typical graphs are shown in Figure 1. In this figure, as well as often in later discussions, we freely identify circle maps with maps on the unit interval with periodic boundary conditions. Thus the derivative of a map can be thought of in an elementary sense, without reference to the smooth manifold structure of the circle.

The first class we shall study are the tip maps (the sawtooth maps with sharp teeth), a terminology whose motivation will become apparent when we introduce the second class. The tip maps are defined by the following piecewise linear forms:

$$
t_{\Omega, S ; \delta}(x)=\left\{\begin{array}{clr}
(S x+\Omega)_{1} & \text { if } & 0 \leq x \leq \frac{1-\delta}{2}, \\
\left(s\left(x-\frac{1}{2}\right)+\frac{1}{2}+\Omega\right)_{1} & \text { if } & \frac{1-\delta}{2} \leq x \leq \frac{1+\delta}{2} \\
(S(x-1)+\Omega+1)_{1} & \text { if } & \frac{1+\delta}{2} \leq x \leq 1,
\end{array}\right.
$$

where the small slope $s$ is given by $s=\frac{[1-S(1-\delta)]}{\delta}<S$, and $S \geq 1$ is the large slope. We shall denote by $I_{\delta}$ the interval $\left[\frac{1-\delta}{2}, \frac{1+\delta}{2}\right]$ where the slope is $s$. Figures 1 a-c display graphs of tip maps for three sets of parameter values. Notice that the tip maps depend on three parameters: the bare frequency parameter, $\Omega$ (corresponding to the parameter $a$ in the usual parametrization of the standard family), the large slope parameter, $S$ (corresponding to the strength of the nonlinear coupling $b$ in the standard family), and a parameter $\delta$, which determines the width of the piece with slope $s$. The tip map (thought of as being deformed from a rotation) becomes non-invertible when the small slope crosses zero (from above); when this small slope is exactly zero, the map is termed critical and the value of the large slope at criticality is $S_{c}(\delta)=\frac{1}{(1-\delta)}$, so that $\delta=\frac{S_{c}(\delta)-1}{S_{c}(\delta)}$. Recall that the standard circle map (and more generally a smooth circle map) is critical when it is monotonic but has zero derivative at (at least) one point. We shall denote by $T_{\omega, S ; \delta}$, with $(\omega)_{1}=\Omega$, a lift $_{A-1}$ of $t_{\Omega, S ; \delta}$. 
The second class we call the plateau maps. These maps are defined by

$$
p_{\Omega, U ; \delta}(x)=\left\{\begin{array}{ccrr}
\left(S_{c} x+\Omega\right)_{1} & \text { if } & 0 \leq x \leq \frac{1-\delta}{2}+\frac{H}{S_{c}}, \\
\left(\frac{1}{2}+H+\Omega\right)_{1} & \text { if } & \frac{1-\delta}{2}+\frac{H}{S_{c}} \leq x \leq \frac{1}{2}-\frac{H}{S_{c}} \\
\left(S_{c}\left(\frac{1}{2}-x\right)+\frac{1}{2}+\Omega\right)_{1} & \text { if } & \frac{1}{2} \frac{H}{S_{c}} \leq x \leq \frac{1}{2}+\frac{H}{S_{c}} \\
\left(\frac{1}{2}-H+\Omega\right)_{1} & \text { if } & \frac{1}{2}+\frac{H}{S_{c}} \leq x \leq \frac{1+\delta}{2}-\frac{H}{S_{c}} \\
\left(S_{c}(x-1)+1+\Omega\right)_{1} & \text { if } & \frac{1+\delta}{2}-\frac{H}{S_{c}} \leq x \leq 1,
\end{array}\right.
$$

for $U \geq S_{c}=S_{c}(\delta)$, with $H=U-S_{c}$, and by $p_{\Omega, U ; \delta}=t_{\Omega, U ; \delta}$ for $U \leq S_{c}$. The maximum value of $H$ is $H_{\max }(\delta)=\frac{S_{c} \delta}{4}$. Figures 1 a,b,d illustrate three members of this family. Set $U_{\max }=S_{c}+H_{\max }$; the graph of $p_{\Omega, U ; \delta}$ for $S_{c} \leq U \leq U_{\max }$ is obtained from the graph of $p_{\Omega, U_{\max } ; \delta}$ by cutting with two horizontal lines. We shall denote by $P_{\omega, U ; \delta}$, with $(\omega)_{1}=\Omega$, a lift of $p_{\Omega, U ; \delta}$.

\section{Parameter spaces.}

We first choose the parameter space for the maps $t_{\Omega, S ; \delta}$ :

- clearly $\Omega$ can be thought of as an element of $\mathbb{T}$, or of $[0,1)$,

- since $s>1$ (and $s>S$ ) when $S<1, S$ can be chosen in $[1,+\infty)$, - since we want to consider only continuous maps, we have to take $\delta$ in $[0,1)$.

Notice then that $S$ has to be equal to 1 when $\delta=0$, and that $s=1$ when $S=1$, so that the value of $\delta$ is irrelevant when $S=1$. It follows that the value 0 of $\delta$ can be omitted. In formal mathematical notation, we introduce the equivalence relation $\sim$ on $\mathbb{T} \times[1,+\infty) \times(0,1)$, defined by $\left(\alpha, 1, \delta_{1}\right) \sim\left(\alpha, 1, \delta_{2}\right)$ for all $\delta_{1}$ and $\delta_{2}$, and by equality when the second coordinate of the triplet is other than 1 . Then the parameter space for the tip maps can be described as

$$
\mathcal{P}_{t}^{\prime}=(\mathbb{T} \times\{1\} \times\{0\} \sqcup \mathbb{T} \times[1,+\infty) \times(0,1)) / \sim,
$$

where the symbol $\sqcup$ stands for disjoint union. However, we find it more convenient to forget the quotient operation, and consider the parameter space

$$
\mathcal{P}_{t}=\mathbb{T} \times\{1\} \times\{0\} \sqcup \mathbb{T} \times[1,+\infty) \times(0,1) .
$$


We shall study the bifurcations and aspects of the dynamics throughout $\mathcal{P}_{t}$, by splitting this 3-dimensional space into disjoint cylinders $\delta=$ constant (see Figure 2). Other 2dimensional subspaces of $\mathcal{P}_{t}$ are worth noticing, and we now list some of them:

- the critical surface $S=S_{c}(\delta)=\frac{1}{1-\delta}$ is the locus of parameter triples corresponding to critical maps. It is the disjoint union of the critical lines in the cylinders $\delta=$ constant.

- The surface $S=\frac{1}{1-2 \delta}$, where $S=-s$ : this surface is everywhere supercritical, except at $S=1$, and the topological entropy $y_{A-4}$ there is trivially equal to $\log (S)$.

- The surface $\delta=\frac{1}{2}$, to which the previous one is asymptotic as $S \rightarrow \infty$, and was the subject of some numerical studies reported in $[\mathrm{YH}]$ and $[\mathrm{UC}]$. As discussed in $[\mathrm{UC}]$, the case $\delta=\frac{1}{2}$ arises naturally for the two-slope case when one is considering successive $n$ slope piecewise linear approximations - i.e., linear splines - to the standard family of circle maps.

- The surface $\Omega=0$ is studied in [AM2]: these authors reparametrize the part of this surface above its critical line by $S$ and $\sigma=-s$ (with our notations), and show among other things that the entropy strictly increases with either $S$ or $\sigma$, the other parameter being kept fixed. For related results, see for instance $[\mathrm{MV}],[\mathrm{Ga}],[\mathrm{GaT}],[\mathrm{GMT}]$ and references therein.

The parameter space $\mathcal{P}_{t}$ has universal cover

$$
\mathbf{P}_{t}=\mathbb{R} \times[1,+\infty) \times(0,1),
$$

with fundamental domain

$$
[0,1) \times[1,+\infty) \times(0,1)
$$

This fundamental domain is partitioned in slices $[0,1) \times[1,+\infty) \times\{\delta\}$ for $\delta \in(0,1)$, and we shall use such slices to draw several figures of this paper. In each slice, the line $S=1$ is called the rotation line, and as discussed previously, all rotation lines represent the same set of maps, to wit the rigid rotations. $\mathbf{P}_{t}$ is also the parameter space for the lifts $T_{\omega, S ; \delta}$ of tip maps. For any $\delta \in[0,1)$, we shall denote by $\mathbf{F}_{t ; \delta}$ the two-parameter family of lifts $T_{\omega, S ; \delta}$. The parameter space $\mathcal{P}_{p}$ for the maps $p_{\Omega, S ; \delta}$ coincides with $\mathcal{P}_{t}$ under and up to the critical surface. Above this surface, $U$ is limited to the range $\left[S_{c}(\delta), S_{c}(\delta)+H_{\max }(\delta)\right]$. Hence

$$
\mathcal{P}_{p}=\left\{(\Omega, U, \delta) \mid \Omega \in \mathbb{T}, \delta \in[0,1), U \in\left[1, S_{c}(\delta)+H_{\max }(\delta)\right]\right\}
$$


As for $\mathcal{P}_{t}$, we shall study the bifurcations and aspects of the dynamics throughout $\mathcal{P}_{p}$, by splitting this 3 -dimensional space into disjoint cylinders $\delta=$ constant. We did not find any other 2 dimensional subspaces of $\mathcal{P}_{p}$ worth noticing, but we remark that the onedimensional subspaces corresponding to fixed $\delta$ and $\Omega$ are stunted families $_{B}$ beyond the critical point. The universal cover of $\mathcal{P}_{p}$ is

$$
\left\{(\omega, U, \delta) \mid \omega \in \mathbb{R}, U \in\left[1, S_{c}(\delta)+H_{\max }(\delta)\right], \delta \in[0,1)\right\}
$$

with fundamental domain

$$
\mathbf{P}_{p}=\left\{(\omega, U, \delta) \mid \omega \in[0,1), U \in\left[1, S_{c}(\delta)+H_{\max }(\delta)\right], \delta \in[0,1)\right\}
$$

This fundamental domain is partitioned in slices $[0,1) \times\left[1, S_{c}(\delta)+H_{\max }(\delta)\right] \times\{\delta\}$ for $\delta \in[0,1)$ and, like for tip maps, we shall use such slices for drawing purposes. $\mathbf{P}_{p}$ is also the parameter space for the lifts $P_{\omega, U ; \delta}$ of plateau maps. For any $\delta \in[0,1)$, we shall denote by $\mathbf{F}_{p ; \delta}$ the two-parameter family of lifts $P_{\omega, U ; \delta}$. We have represented $\mathcal{P}_{t}$ and $\mathcal{P}_{p}$ in Figures 2 and 3.

\section{Monotone bounds.}

With the natural partial order on real maps (see Appendix A-2), the monotone bounds of a real function $F$ are the monotone upper bound $F^{+}$, defined as the smallest monotone function greater than or equal to $F$, and the monotone lower bound $F^{-}$, defined as the largest monotone function smaller than or equal to $F$ : see Appendix A-2 for a review on monotone bounds. All monotone bounds for the tip and plateau maps above or on the critical line are critical maps of our families. More specifically, the circle maps obtained by projecting the monotone bounds read:

$$
\begin{aligned}
& t_{\Omega, S ; \delta}^{+}(x)=t_{\Omega+\frac{S-1-S \delta}{2}, S ; \frac{S-1}{S}}\left(\left(x-\frac{S-1-S \delta}{2 S}\right)_{1}\right), \\
& t_{\Omega, S ; \delta}^{-}(x)=t_{\Omega-\frac{S-1-S \delta}{2}, S ; \frac{S-1}{S}}\left(\left(x+\frac{S-1-S \delta}{2 S}\right)_{1}\right),
\end{aligned}
$$

and

$$
p_{\Omega, U ; \delta}^{+}(x)=t_{\Omega+\delta\left(U-\frac{1}{1-\delta}\right), \frac{1}{1-\delta} ; \delta}\left((x-U(1-\delta)+1)_{1}\right)
$$




$$
p_{\Omega, U ; \delta}^{-}(x)=t_{\Omega+\delta\left(-U+\frac{1}{1-\delta}\right), \frac{1}{1-\delta} ; \delta}\left((x+U(1-\delta)-1)_{1}\right) .
$$

Remark. For the families of maps we consider in this paper, the critical maps, the monotone bounds above the critical line, and the $F_{\omega}$ 's of Theorem B in Appendix A-2, all can be reinterpreted using the following construction when the rotation number is in $(0,1)$ (see Figure 4 and [Ve1-Ve2]).

- Start with the unit square, and draw the first diagonal and the lines $\Delta_{0}: y=S x$ and $\Delta_{1}: y=S(x-1)+1$, for any $S>1$.

- Choose any $A \leq \frac{1}{S}$, and draw the square $Q_{A}$ with corners $(A, A)$ and $\left(A+\frac{S-1}{S}, A+\frac{S-1}{S}\right)$.

- The pieces of $\Delta_{0}$ and $\Delta_{1}$ in $Q_{A}$ can be completed to the graph of a critical circle map, where the circle has length $\frac{S-1}{S}$.

- Now, varying A monotonically, one gets a parametrization of either the critical maps with slope $S$, one of the families of monotone bounds described previously, or the family of the $F_{\omega}$ 's of Theorem B for some map with the given $S$.

- Using the kneading theory of nicely ordered $_{A-2}$ periodic orbits (see [STZ] and references therein for a recent review of this centuries old subject [Be], [Ch], [Sm], [Ma], $[\mathrm{MH}]$ ), one can check that the $A$-interval corresponding to any rotation number of the form $\frac{p}{q}$ has length $\frac{(S-1)^{2}}{S\left(S^{q}-1\right)}$ (see [Ve3] for details).

\section{Some special sets in function and parameter space}

Let $C^{0}(\mathbb{R})$ be the space of continuous degree one lifts endowed with the sup norm. Following [Boyl] and [MaT1] (which both extended to degree one circle maps some pieces of the theory developed in $[\mathrm{Ar}]$ for homeomorphisms) for each real number $\omega$ we define two subsets of $C^{0}(\mathbb{R})$ as follows, where $I(F)$ denotes the rotation interval $_{A-2}$ :

$$
\mathcal{A}_{\omega}=\left\{F \in C^{0}(\mathbb{R}) \mid \omega \in I(F)\right\}
$$

and

$$
\mathcal{L}_{\omega}=\left\{F \in C^{0}(\mathbb{R}) \mid\{\omega\}=I(F)\right\}
$$


It is easy to prove that both sets are path-connected for any $\omega \in \mathbb{R}$. In words, $\mathcal{L}_{\omega}$ is the subset in which there is a unique rotation number $\omega$, whereas $\mathcal{A}_{\omega}$ is the set in which $\omega$ is among the (many, possibly a continuum of) rotation numbers.

For $\delta \in[0,1)$, let $\mathbf{F}_{t ; \delta}$ and $\mathbf{F}_{p ; \delta}$ be the two-parameter families of lifts corresponding respectively to tip maps and to plateau maps (see $\S 3$ ). For each real number $\omega$, and $u \in\{t, p\}$, we define

$$
A_{\omega, u}=\mathcal{A}_{\omega} \cap \mathbf{F}_{u, \delta},
$$

and

$$
L_{\omega, u}=\mathcal{L}_{\omega} \cap \mathbf{F}_{u, \delta} .
$$

Hence the $A_{\omega, u}$ 's and the $L_{\omega, u}$ 's are subsets of two- dimensional spaces.

To simplify the language and the notations, we shall identify sets of standard lifts with the corresponding regions in parameter space: the context should tell which space we mean (parameter space or function space), when the distinction is relevant. For statements where the subscript $u$ could be either $t$ or $p$, we usually suppress it. Thus $A_{\omega}$ stands for " $A_{\omega, t}$ or $A_{\omega, p}$ " and so on.

For the region $A_{\omega}$, we have a decomposition into a disjoint union

$$
A_{\omega}=A_{\omega}^{-} \sqcup A_{\omega}^{o} \sqcup A_{\omega}^{+},
$$

where "-" stands for the subcritical region in which $F \in \mathbf{F}_{u, \delta}$ is strictly increasing; "o" stands for the critical line for which $F \in \mathbf{F}_{u, \delta}$ is increasing but has zero derivative at at least one point; and "+" stands for the supercritical region, where $F \in \mathbf{F}_{u, \delta}$ is non-monotonic. Similarly, we can write

$$
L_{\omega}=L_{\omega}^{-} \sqcup L_{\omega}^{o} \sqcup L_{\omega}^{+}
$$

and Theorem 3 will tell us that $L_{\omega}^{+}=\emptyset$ when $\omega$ is an irrational number. Defining $A^{\ominus}=$ $A^{o} \cup A^{-}$and $A^{\oplus}=A^{o} \cup A^{+}$, we can also write

$$
A_{\omega}=A_{\omega}^{\ominus} \sqcup A_{\omega}^{+}=A_{\omega}^{-} \sqcup A_{\omega}^{\oplus} .
$$

Similarly, with $L^{\ominus}=L^{o} \cup L^{-}$and $L^{\oplus}=L^{o} \cup L^{+}$, we will write

$$
L_{\omega}=L_{\omega}^{\ominus} \sqcup L_{\omega}^{+}=L_{\omega}^{-} \sqcup L_{\omega}^{\oplus} .
$$




\section{General results concerning the sets $A_{\omega}$ and $L_{\omega}$}

We begin with three general results concerning the "shape" and the boundaries of the sets $A_{\omega}$ and $L_{\omega}$ in parameter space. Since these results hold not only for our piecewise linear families, but also for the standard family (and with similar proofs, see [Boyl], [MaT1] and [ORSS]), we shall be fairly brief. Non-expert readers wishing to follow closely should consult the references and work through our arguments in detail.

Theorem 1: Connectedness of the $A_{\omega}$ 's. $\forall \omega \in \mathbb{R}, A_{\omega}$ is a connected and simply connected subset of $\mathbf{F}_{\delta}$.

Proof of Theorem 1. This follows directly from the continuity of the bounds of the rotation interval, which in turn is a direct consequence of the combination of Theorems $\mathrm{A}(\mathrm{ii})$ and $\mathrm{B}(\mathrm{i})$, and of the monotonicity of these bounds as a function of $\omega$, which comes from Corollary A' and Theorem B(i) in Appendix A-2.

(Q.E.D. Theorem 1.)

Let us say that a set of curves in $\mathbb{R} \times \mathbb{R}^{+}$is an $L$-set of curves if for some $K$, and for all curves in the set, the first coordinate is a uniform Lipschitz function of the second one, with Lipschitz constant $K$. Once such a $K$ is determined, we use the term $L$-set of curves with Lipschitz constant $K$. (Recall that $g$ is a Lipschitz function with Lipschitz constant $K$ if for all $x$ and $y,|g(x)-g(y)| \leq K|x-y|$.)

\section{Theorem 2: A uniform Lipschitz property.}

i) - The left and right boundaries, $A_{\omega}^{l}$ and $A_{\omega}^{r}$, of the $A_{\omega}$ 's in $\mathbb{R} \times \mathbb{R}^{+}$form an $L$ - set of curves.

ii) - The collection of the sets

$$
\begin{aligned}
& B_{\omega}^{l}=\lim _{\theta \rightarrow \omega^{+}} A_{\theta}^{l}, \\
& B_{\omega}^{r}=\lim _{\theta \rightarrow \omega^{-}} A_{\theta}^{r},
\end{aligned}
$$

form an $L$-set of curves. 
iii) - For any $\omega$

$$
\begin{aligned}
& A_{\omega}^{l}=\lim _{\theta \rightarrow \omega^{-}} A_{\theta}^{l}, \\
& A_{\omega}^{r}=\lim _{\theta \rightarrow \omega^{+}} A_{\theta}^{r} .
\end{aligned}
$$

- For $\omega$ irrational

$$
\begin{aligned}
& A_{\omega}^{l}=B_{\omega}^{l}, \\
& A_{\omega}^{r}=B_{\omega}^{r} .
\end{aligned}
$$

Proof of Theorem 2. We prove i) first, and first consider the tip case, which covers the plateau case below and up to the critical line: this proof is exactly as in the standard family (see [Boyl], [EKT]). In fact we prove statement i) only for $A_{\frac{p}{q}}^{l}$ since the remaining cases can be treated in an analogous way.

Claim. If $T_{\omega, S ; \delta} \in A_{\frac{p}{q}}^{l}$, the vertical cone in $\mathbb{R} \times \mathbb{R}^{+}$, with vertex at $T_{\omega, S ; \delta}$, and boundaries made by lines with slopes $\frac{2}{1-\delta}$ and $-\frac{2}{1-\delta}$, contains $A_{\frac{p}{q}}^{l}$.

Proof of the Claim. A direct computation yields

$$
\forall \epsilon>0 \quad, \forall \mu \in\left[0, \frac{2 \epsilon}{1-\delta}\right] \quad T_{\omega-\epsilon, S \pm\left(\frac{2 \epsilon}{1-\delta}-\text { Claim }\right) ; \delta} \leq T_{\omega, S ; \delta},
$$

and

$$
\forall \epsilon>0 \quad, \forall \epsilon^{\prime}>0 \quad, \forall \mu \in\left[0, \frac{2 \epsilon}{1-\delta}\right] \quad T_{\omega-\epsilon-\epsilon^{\prime}, S \pm\left(\frac{2 \epsilon}{1-\delta}-\mu\right) ; \delta} \leq T_{\omega-\epsilon^{\prime}, S ; \delta} .
$$

Consequently, using the continuity of the rotation number of $T_{\omega, S ; \delta}^{+}$as a function of the parameters, and its monotonicity as a function of $\omega$, we have

$$
\rho\left(T_{\omega-\epsilon, S \pm\left(\frac{2 \epsilon}{1-\delta}-\mu\right) ; \delta}^{+}\right) \leq \frac{p}{q}
$$

and

$$
\rho\left(T_{\omega-\epsilon-\epsilon^{\prime}, S \pm\left(\frac{2 \epsilon}{1-\delta}-\mu\right) ; \delta}^{+}\right)<\frac{p}{q} .
$$

Similarly

$$
\forall \epsilon>0 \quad, \forall \mu \in\left[0, \frac{2 \epsilon}{1-\delta}\right] \quad T_{\omega+\epsilon, S \pm\left(\frac{2 \epsilon}{1-\delta}-\mu\right) ; \delta} \geq T_{\omega, S ; \delta}
$$


implies

$$
\rho\left(T_{\omega+\epsilon, S \pm\left(\frac{2 \epsilon}{1-\delta}-\mu\right) ; \delta}^{+}\right) \geq \frac{p}{q} .
$$

The claim then follows from the three inequalities on $\rho$.

(Q.E.D. Claim.)

The proof for the plateau case above the critical line is even easier: from the formula given in $\S 4$, it readily follows that for any $\delta$, and above the critical line, all curves $A_{\omega}^{l}$ and $B_{\omega}^{l}$ are parallel straight lines with slope $-\frac{1}{\delta}$ in the $(\omega, U)$ plane, while the curves $A_{\omega}^{r}$ and $B_{\omega}^{r}$ are parallel straight lines with slope $\frac{1}{\delta}$, which means that these curves above the critical line form an $L$-set of curves with Lipschitz constant $\delta$.

Statements ii) and iii) follow from i) combined with Theorems A(ii) and B(i) in Appendix A-2.

(Q.E.D. Theorem 2.)

Theorem 3: The Nature of $L_{\omega}$ and $A_{\omega}$ for irrational $\omega$. With $\mathbb{Q}$ indicating the set of rationals, we have

-(i). $\forall \omega \in(\mathbb{R} \backslash \mathbb{Q}), L_{\omega}=A_{\omega}^{\ominus}$ in $\mathbf{F}_{\delta}$,

-(ii). $\forall \omega \in(\mathbb{R} \backslash \mathbb{Q}), L_{\omega}$ is a Lipschitz curve joining $S=1$ to $S=S_{c}$ in the parameter space.

-(iii). The intersection of $A_{\omega}$ with any horizontal line above the critical line in $\mathbf{F}_{\delta}$, is an interval of positive length.

\section{Proof of Theorem 3.}

-(i). This statement can be reformulated as the following

Claim Above the critical line, one cannot have $\rho\left(F^{-}\right)=\rho\left(F^{+}\right) \notin \mathbb{Q}$.

The proof of this claim goes as for the standard family (see $[\mathrm{BlF}]$ and $[\mathrm{CGT}]$ ) and relies on classical results recalled in Appendix A-2: we first remark that there exist distinct $C^{2}$ smooth lifts $F_{0}$ and $F_{1}$ such that, $\forall x \in \mathbb{R}, F^{-}(x) \leq F_{0}(x) \leq F_{1}(x) \leq F^{+}(x)$. If the claim is false, by Theorem $\mathrm{A}(\mathrm{i}), \rho\left(F_{0}\right)=\rho\left(F_{1}\right)=\rho\left(F^{+}\right) \notin \mathbb{Q}$, so that $F_{0}$ (and $F_{1}$ ) has a dense orbit by Denjoy Theorem. Hence the claim follows from Theorem A(iii). -(ii) follows from Theorem A(i)-(ii) and Theorem 2. 
-(iii) follows from (i) and Theorems A(ii) and B(i).

(Q.E.D. Theorem 3.)

\section{Results for $A_{\omega}$ and $L_{\omega}$ below and on the critical line.}

Before reporting our technical results, let us provide a few introductory comments to guide the reader. Below and on the critical line, the $L_{\omega}$ 's and the $A_{\omega}$ 's coincide, i.e. $L_{\omega}^{\ominus}=A_{\omega}^{\ominus}$. By the uniqueness of the rotation number for fomeomorphisms $s_{A-2}$, these sets form a partition of the parameter space. The topology and geometry of the $L_{\omega}^{\ominus}$ 's with irrational $\omega$ is described in Theorem 3(ii). For the rational case, the topology of $L_{p / q}^{\ominus}$ is covered by Theorem 4; we relegate the explicit computations (essential for constructing figures to scale) to Appendix C. In the statement of Theorem 4, a node means a point in $L_{p / q}^{\ominus}$ above the $S=1$ line of trivial rotations (i.e., we must have $S>1$ ), at which point the left and right boundaries of $L_{p / q}^{\ominus}$ coincide, so that taking out this point would disconnect $L_{p / q}^{\ominus}$. The question of how the rational and the irrational $L_{\omega}^{-}$'s "share" the Lebesgue measure of the parameter space below the critical line is still open: numerical results are reported in Appendix D (see also the last remarks at the end of the present section). The description of behavior on the critical line is in Theorem 5, which slightly improves on the general result of [Boyd] specialized to our case (see also [Ve3]).

Theorem 4: Topology of the $L_{p / q}^{\ominus}$ : the "Sausage" Structure. In $\mathbf{F}_{\delta}, \forall q \in \mathbb{Q}, L_{p / q}^{\ominus}=$ $A_{p / q}^{\ominus}$ has $\lceil\delta q\rceil-1$ nodes, where $\lceil x\rceil$ is the smallest integer greater than or equal to $x$. Hence, the interior of $L_{p / q}^{\ominus}$ has $\lceil\delta q\rceil$ connected components, each of which is simply connected.

Proof of Theorem 4. The graph of the $q^{\text {th }}$ iterate of any map $g$ in $L_{p / q}^{\ominus}$ intersects the diagonal at the periodic points of the map (see Appendix A-2). If the intersections are transversal, intersections occur for the graph of the $q^{\text {th }}$ iterate of any map close enough to $g$; this leads to the finite widths of the mode-locking intervals (Arnold tongues). The only way that any small (additive) perturbation of $g$ can generate no intersection of the graph of its $q^{\text {th }}$ iterate with the diagonal is if the $q^{\text {th }}$ iterate of $g$ is the identity; hence nodes can only occur in this case. It thus remains to count how often such situations occur in $L_{p / q}^{\ominus}$. 
For $S=1$, all maps are rotations, so in $L_{p / q}$, the number of points of the periodic orbit to which $\frac{1-\delta}{2}$ belongs, and which are in $\left[\frac{1-\delta}{2}, \frac{1+\delta}{2}\right]$, is $\lceil\delta q\rceil$. For $S=S_{c}$, on the left boundary of $L_{p / q}^{o}, \frac{1-\delta}{2}$ is the only point of $\left[\frac{1-\delta}{2}, \frac{1+\delta}{2}\right]$ in a periodic orbit. By continuity, going down from $S_{c}$ to $S$ on the left boundary of $L_{p / q}^{\ominus}$, where $\frac{1-\delta}{2}$ is a point of the unique periodic orbit, $\lceil\delta q\rceil-1$ points of the orbit of $\frac{1-\delta}{2}$ have to get in $\left[\frac{1-\delta}{2}, \frac{1+\delta}{2}\right]$. Each time one point crosses $\frac{1+\delta}{2}$, the $q^{\text {th }}$ iterate of the map $t_{\Omega, S ; \delta}$ is the identity map $I d$, hence, we get a node of $L_{p / q}^{\ominus}$. It only remains to show that each crossing occurs once. But if $m$ points of the orbit of $\frac{1-\delta}{2}$ belong to $\left[\frac{1-\delta}{2}, \frac{1+\delta}{2}\right]$, the equality

$$
t_{\Omega, S ; \delta}^{q}=I d
$$

implies

$$
s^{m-1} S^{q+1-m}=1,
$$

or

$$
\left[\frac{1-S(1-\delta)}{\delta}\right]^{m-1} S^{q+1-m}=1
$$

and elementary algebra shows this equation in $S$ has at most one solution in $\left(1, \frac{1}{1-\delta}\right)$.

(Q.E.D. Theorem 4.)

\section{Remarks.}

- The fact that powers of piecewise linear circle homeomorphisms can be the identity, which is the core of the sausage shape of the $L_{p / q}^{-}$'s has, been known for a long time: for a deep result using this fact, see [He2]. However the Arnold sausages have not previously been described in print in a quantitative way, except in $[\mathrm{YH}]$, which considers without giving a proof the case of $\delta=\frac{1}{2}$ ( we thank Leon Glass for pointing out this reference to us).

- As indicated in the introduction, the mode- or frequency-locking phenomenon, first described (in the context of phase-locking or synchronization in the 1:1 region) by Christian Huyghens in the context of pendulum clocks hanging on the same wall, refers to the fact that generically, coupled non-linear oscillators beating at rationally related frequencies will continue to do so, with the same rational relation, under a small enough perturbation. For circle map models of forced oscillators, this corresponds to the non-zero widths of the $L_{p / q}^{-}$'s (or $A_{p / q}^{-}$'s) whenever the non-linearity parameter is not zero (i.e., away from the 
$S=1$ line of trivial rotations). For generic smooth circle maps, and restricting to the real line of entire nonlinear functions, no iterate can be the identity [He2], which prevents any line in parameter space not intersecting the line of pure rotations from crossing the $L_{p / q}$ 's at a single point and hence insures frequency locking. Thus, by preventing frequency locking, the sausage shape described in Theorem 4 can be viewed as a pathology of the piecewise-linear models: typically, one rather tries to ensure frequency locking in physical systems and assumes it in mathematical analyses. For instance, in [Boyl], it is assumed that, except for rotations, no iterate of the maps considered can be the identity. In the concluding section, we shall discuss one physical system in which the "Arnold sausage" structure has been observed.

Theorem 5: Likelihood of irrational rotation numbers for $S=S_{c}$. For any $\delta \in(0,1)$, the set $E_{\delta}=\left(\Omega \mid \rho\left(t_{\Omega, S_{c}(\delta) ; \delta}\right) \notin \mathbb{Q}\right)$ has zero box dimension $d_{B}(\delta)$.

Remark. It was previously known that the set $E_{\delta}$ has zero Lebesgue measure and zero Hausdorff dimension ([Boyd], [Ve3]). For generic families of smooth enough maps with at most two critical points, such as the standard family, it is known that irrational rotation numbers correspond to zero Lebesgue measure $[\mathrm{Sw}]$, and in fact form a set of Hausdorff dimension smaller than 1 on the critical line [Kh]. Furthermore, there is numerical evidence that this dimension is positive and universal (with the degeneracy (i.e., the order) of the critical point as a modulus) [JBB1], [JBB2].

Proof of Theorem 5. Fix $\delta \in(0,1)$. For $r>0$, let $K_{r}(\delta)$ be the number of $L_{p / q}^{o}$ 's of length $\geq r$. Since we know from [Boyd] that the set $E_{\delta}$ has zero Lebesgue measure, we get from [Tri] that the box dimension $d_{B}(\delta)$ of $E_{\delta}$ is:

$$
d_{B}(\delta)=\limsup _{r \rightarrow 0} \frac{\log K_{r}(\delta)}{\log (1 / r)}
$$

From the Remark in $\S 4$

$$
\left|L_{p / q}^{o}\right|=\frac{\left(S_{c}(\delta)-1\right)^{2}}{S_{c}(\delta)\left(\left(S_{c}(\delta)\right)^{q}-1\right)},
$$

where one only considers the $L_{p / q}^{o}$ 's with $q>1$, hence

$$
d_{B}(\delta)=\limsup _{r \rightarrow 0} \frac{\log K_{r}(\delta)}{\log (1 / r)}=\limsup _{q \rightarrow \infty} \frac{\log K_{\left|L_{p / q}^{o}\right|}(\delta)}{\log \left[\frac{S_{c}(\delta)\left(\left(S_{c}(\delta)\right)^{q}-1\right)}{\left(S_{c}(\delta)-1\right)^{2}}\right]}=0,
$$


since $S_{c}>1$ and $K_{\left|L_{p / q}^{o}\right|}$ grows as a polynomial in $q$.

(Q.E.D. Theorem 5.)

\section{Remarks.}

- Using the above formula for $\left|L_{p / q}^{o}\right|$ and the well known identity [HW]

$$
\sum_{k=1}^{\infty} \frac{x^{k}}{1-x^{k}} \phi(k)=\frac{x}{(1-x)^{2}}
$$

where $\phi(n)$ is Euler's totient function, which counts the integers smaller than $n$ and coprime with $n$, it is easy to check directly that

$$
\sum_{q=2}^{\infty}\left|L_{p / q}^{o}\right|=\frac{1}{S_{c}},
$$

so that by the Remark in $\S 4$, the set $E_{\delta}$ has zero Lebesgue measure: this is essentially the proof given in [Ve3]. The proof given in [Boyd] covers a much wider class of maps.

- It was asserted in [UTGC] that $\forall S<S_{c}$ and $\forall \delta \in(0,1), \mu\left(\Omega \mid \rho\left(t_{\Omega, S ; \delta}\right) \notin \mathbb{Q}\right)>0$, where $\mu(E)$ is the Lebesgue measure of the set $E$. However, the Lemma (not only its proof) we thought we had to prove this measure property is wrong. On the other hand we have some numerical results and remarks suggesting that the measure property is true (see Appendix D), in contrast to a rigorous result in [VK] establishing zero measure of $E$ for piecewise smooth circle maps with a single singular point; the resolution of this seeming contradiction is simply that our tip maps have two singular points.

- It is also worth noting that, for each $\delta \in(0,1)$, there are countably many rational curves $l_{(n, m)}$, with $(n, m) \in \mathbb{Z}^{+} \times \mathbb{N}$, in the $(\omega, S)$ parameter space, which cross each $L_{p / q}$ at at most one point. Clearly, irrational rotation numbers correspond to a set of full measure on these curves. With $T_{\omega, S ; \delta}$ standing for the lift of $t_{\Omega, S ; \delta}$ such that $T_{\omega, S ; \delta}(0) \in[0,1)$, the curve $l_{(n, m)}$ is the solution of

$$
T_{\omega, S ; \delta}^{n}\left(\frac{1-\delta}{2}\right)=\frac{1+\delta}{2}+m
$$

Some of these curves were drawn in $[\mathrm{YH}]$ in the case when $\delta=\frac{1}{2}$. In our Appendix $\mathrm{C}$, two such curves are drawn for the case of $\delta=0.6$ (see Fig. C16). 
- The measure property we conjecture to be true for our piecewise linear maps is known to hold for the standard family, as a particular case of a more general and quite deep result of Michael Herman [He1].

\section{Maps with given rotation interval.}

The main result in this section is Theorem 8 which describes the sets of maps with given rotation interval. The other Theorems describe structure properties of maps satisfying certain conditions, which are useful to prove Theorem 8 or the results in the next section about the boundary of chaos. Some of the structure properties are related to nicely ordered orbits and related concepts, as reviewed in Appendix A-2. The reader is reminded that $\mathbf{F}_{\delta}$ denotes either of the tip or plateau map families (see $\S 5$ ).

Theorem 6: Uniqueness of the pair of well-ordered periodic orbits in $A_{p / q}^{+}$.

- Any $F_{\omega, T ; \delta} \in \mathbf{F}_{\delta}$ in the interior of $A_{p / q}^{+}$has exactly two $p / q$-ordered orbits.

- Exactly one of these orbits, denoted $\mathbf{O}^{\prime} \frac{p}{q}$, is contained in the regions where $F_{\omega, T ; \delta}$ is increasing.

- There exists $T_{c}^{\prime}\left(\omega, \mathbf{F}_{\delta}\right)>S_{c}$ such that for each $S<T_{c}^{\prime}\left(\omega, \mathbf{F}_{\delta}\right), \mathbf{O}^{\prime} \frac{p}{q}$ is unstable and the other $p / q$-ordered orbit is stable.

- There is a single $p / q$-ordered orbit, also denoted $\mathbf{O}^{\prime} \frac{p}{q}$ for $F_{\omega, T ; \delta}$ on the boundary of $A_{p / q}^{+}$: this orbit contains the lifts of at least one of the singular points of $f_{\Omega, T ; \delta}$ with $\Omega=(\omega)_{1}$.

\section{Proof of Theorem 6.}

Using, e.g., the Remark in $\S 4$ or the fact that $S>1$, the proof is an exercise left to the studious reader; as a hint, consider the lift of the $q^{\text {th }}$ iterate of the map zigzagging back and forth across the line $y=x$.

(Q.E.D. Theorem 6.)

Let $\mathbf{O}_{\frac{P}{Q}}=\left\{P_{0}, P_{1}, \ldots, P_{Q-1}\right\}$, be the projection of $\mathbf{O}^{\prime} \frac{p}{q}$ onto the circle, where we have set $f_{\Omega, T ; \delta}\left(P_{j}\right)=P_{(j+1)_{Q}}$, and $\frac{P}{Q}=\left(\frac{p}{q}\right)_{1}$. It follows from the properties of $\mathbf{O}_{\frac{P}{Q}}$ that the two critical points $C$ and $K$ of $f_{\Omega, T ; \delta}$ are in an arc $A$ bounded by two successive points $P_{j}$ and $P_{k}$ of $\mathbf{O}_{\frac{P}{Q}}$, which coincide when $Q=1$. Let $P_{j}^{\prime}$ be a lift of $P_{j}, P_{k}^{\prime}$ be the lift of $P_{k}$ 
immediately to the right of $P_{j}^{\prime}$, and let $C^{\prime}$ and $K^{\prime}$ be the lifts of $C$ and $K$ in $\left[P_{j}^{\prime}, P_{k}^{\prime}\right]$. Let $F_{\omega, T ; \delta}$ be the lift of $f_{\Omega, T ; \delta}$ such that $P_{j}^{\prime}$ (and $P_{k}^{\prime}$ ) has rotation number $\frac{p}{q}$ under $F_{\omega, T ; \delta}$, i.e., $\underline{\rho}_{F_{\omega, T ; \delta}}\left(P_{j}^{\prime}\right)=\bar{\rho}_{F_{\omega, T ; \delta}}\left(P_{j}^{\prime}\right)=\frac{p}{q}$. We have

Theorem 7: Boundary behavior. Let $F_{\omega, T ; \delta} \in A_{\frac{p}{q}}^{\oplus}$ and $I\left(F_{\omega, T ; \delta}\right)=[\alpha, \beta]$

i) $\frac{p}{q}=\beta$ if and only if

$$
F_{\omega, T ; \delta}\left(C^{\prime}\right) \leq F_{\omega, T ; \delta}\left(P_{k}^{\prime}\right)
$$

ii) $\frac{p}{q}<\beta$ if and only if

$$
F_{\omega, T ; \delta}\left(C^{\prime}\right)>F_{\omega, T ; \delta}\left(P_{k}^{\prime}\right)
$$

iii) $\frac{p}{q}>\alpha$ if and only if

$$
F_{\omega, T ; \delta}\left(K^{\prime}\right)<F_{\omega, T ; \delta}\left(P_{j}^{\prime}\right)
$$

iv) $\frac{p}{q}=\alpha$ if and only if

$$
F_{\omega, T ; \delta}\left(K^{\prime}\right) \geq F_{\omega, T ; \delta}\left(P_{j}^{\prime}\right)
$$

v) $\frac{p}{q} \in \operatorname{Interior}\left(I\left(F_{\omega, T ; \delta}\right)\right)$ if and only if

$$
F_{\omega, T ; \delta}\left(C^{\prime}\right)>F_{\omega, T ; \delta}\left(P_{k}^{\prime}\right) \quad \text { and } \quad F_{\omega, T ; \delta}\left(K^{\prime}\right)<F_{\omega, T ; \delta}\left(P_{j}^{\prime}\right)
$$

vi) $\left\{\frac{p}{q}\right\}=I\left(F_{\omega, T ; \delta}\right)$ if and only if

$$
F_{\omega, T ; \delta}\left(C^{\prime}\right) \leq F_{\omega, T ; \delta}\left(P_{k}^{\prime}\right) \quad \text { and } \quad F_{\omega, T ; \delta}\left(K^{\prime}\right) \geq F_{\omega, T ; \delta}\left(P_{j}^{\prime}\right) .
$$

Proof of Theorem 7. Statement i) follows readily from Theorem 6 and Theorem B: for $\frac{p}{q}$ to be in the interior of $I\left(F_{\omega, T ; \delta}\right)$, it is necessary and sufficient that $\rho\left(F_{\omega, T ; \delta}^{-}\right)<\frac{p}{q}$ and $\rho\left(F_{\omega, T ; \delta}^{+}\right)>\frac{p}{q}$. Statement ii) is proven similarly, as are iii) and iv). Statements v) and vi) follow from i) through iv).

(Q.E.D. Theorem 7.)

Theorem 8: Maps with given rotation interval. For each $\delta$ and each closed interval $I$, the set of lifts in $\mathbf{F}_{\delta}$ with rotation interval I corresponds to a connected and simply 
connected subset $R_{I}$ of the parameter space $\mathbb{R} \times \mathbb{R}^{+}$or $\mathbb{R} \times\left[0, H_{\max }(\delta)\right]$. More precisely, there exists $K$ as given in the proof of Theorem 2 such that:

- If one endpoint of I is irrational, the second boundary being the same irrational number or a rational number, $R_{I}$ is an arc where the first coordinate is a Lipschitz function, with Lipschitz constant $K$, of the second one.

- If the endpoints of $I$ are distinct irrational numbers, $R_{I}$ is a point.

- When $I=\left\{\frac{p}{q}\right\}, R_{I}$ is bounded by two arcs where the first coordinate is a Lipschitz function, with Lipschitz constant $K$, of the second one; these two arcs intersect at a single point above the critical line and on the rotation line, the other intersections being described in Theorem 4 .

- If the endpoints of $I$ are distinct rational numbers, $R_{I}$ is above the critical line and is bounded by two arcs where the first coordinate is a Lipschitz function, with Lipschitz constant $K$, of the second one. These two arcs have common endpoints and disjoint interiors.

Corollary 8': Topology of $L_{p / q}^{\oplus} \cdot \forall p / q \in \mathbb{Q}, L_{p / q}^{\oplus}$ is a connected and simply connected subset of $\mathbf{F}_{\delta}$.

Remark. At the time [UTGC] was written, Corollary 8' was conjectured to hold for reasonable smooth families (such as the standard family) (see [Boyl] p. 378 and Figure 13, and [MaT1] p.213). This has since been proved, together with most of Theorem 8 , for the standard family: in this family, only the case of a rotation interval with distinct irrational end points is open at this writing: the corresponding region is known to be connected, and conjectured to be a point [EKT].

Proof of Theorem 8. From Theorems 2, 4 and 7, it is sufficient to prove that each $A_{\omega}^{l}$ or $B_{\omega}^{l}$ intersects each $A_{\theta}^{r}$ and $B_{\theta}^{r}$ for $\theta<\omega$, and $B_{\omega}^{r}$ at a unique point above the rotation line when $\omega$ and $\theta$ are both rational or both irrational. In the case of plateau maps, the existence and uniqueness of such intersections follow readily from the proof of Theorem 2 in the plateau case. In the case of tip maps, tongues boundaries above the critical line no longer necessarily correspond to monotonic graphs when the second coordinate is expressed as a function of the first (see Figure 5). Hence we have to prove both existence 
and uniqueness of the crossings. For any $\omega$, when $S$ is large enough, the thinnest strip with sides parallel to the first diagonal and containing the graph of $T_{\omega, S ; \delta}$, is as wide as one wants. Hence:

Lemma 8". For any $\omega$, if $S$ is large enough, $\omega$ is contained in the interior of $I\left(T_{\omega, S ; \delta}\right)$.

Lemma 8" takes care of the existence of the crossings and it remains to control the intersection of $C_{\omega}^{l}$ and $C^{\prime r}{ }_{\theta}^{r}$, with $C$ and $C^{\prime}$ standing throughout the remainder of the proof for either $A$ or $B$. For that, we first recall that, by Theorem 2, the curves whose intersections we consider are graphs of maps from the second coordinate to the first one. Hence, in case of multiple crossings, we would get two maps both with two slopes, say $(S, s)$ and $\left(S^{\prime}, s^{\prime}\right)$ with $S>S^{\prime}>0>s^{\prime}>s$ (or $S^{\prime}>S>0>s>s^{\prime}$ ) both at the intersection of two curves. From kneading theory [MTh], [AM1], we can deduce that these two maps would have the same kneading sequences, hence that the turning points would have identical inverse legal paths (in the sense of $[\mathrm{MSS}]$ ). In the case when $\omega$ and $\theta$ are both rational (see the rational case below) or both irrational (see the irrational case below), we will show that the intersection of $C_{\omega}^{l}$ and ${C^{\prime}}_{\theta}^{r}$ is a unique point. It follows, using Theorem 2-iii), that the intersection of $C_{\lambda}^{l}$ and $C^{\prime r}{ }_{\mu}$ is always a point or an arc. The uniqueness results we can obtain and the limit connectedness of the intersection in all cases is enough to prove the theorem in the tip case. The missing uniqueness results are conjectured to be true but, in the case when one of $\lambda$ and $\mu$ is rational and the other irrational, our methods work only when dealing with maps which have some iterate with slopes everywhere greater than one in absolute value (such cases are treated like the irrational case below).

The rational case. We isolate first the case when $\omega=\frac{p}{q}$ and $\theta=\frac{p^{\prime}}{q^{\prime}}$ are rational numbers. Then the map at the crossing of $C_{\frac{p}{q}}^{l}$ and $C_{\frac{p^{\prime}}{q^{\prime}}}^{r^{r}}$ is a Markov map i.e., the orbits of the turning points are finite, and together give a Markov partition of the circle, whose set of boundaries is forward invariant. The pieces of the partitions for both maps are labeled similarly by kneading theory, as well as all inverses because of the topological conjugacy. Since both absolute values of the slopes for one map are greater than those for the second one, we see that all sufficiently remote backward images of the initial partition for one map get smaller than the similarly labeled pieces for the other map, which is impossible since in 
both cases one has to cover exactly the same circle.

The irrational case. While this question arises naturally in the present context, the result is most easily understood by referring to Theorem 16, which is proved independently in a later section.

From Theorem 16, some iterate of the maps at the crossing of $C_{\omega}^{l}$ and $C^{\prime r}$ have slopes everywhere greater than one in absolute value, and by Corollary 16' and kneading theory, two maps at the intersection of a given pair of curves $C_{\omega}^{l}$ and $C^{\prime r}{ }_{\theta}$ are topologically conjugate. We thus can use a specialization of the result in [GMT], that two piecewise linear maps, one of which has an iterate with slopes greater than one in absolute value, and one of which has all slopes in absolute value greater than the other, cannot be topologically conjugate.

(Q.E.D. Theorem 8.)

Theorem 9: Maps in $L_{\frac{p}{q}}^{+}$. Any map $f \in L_{\frac{p}{q}}^{+}$permutes cyclically $q$ arcs with pairwise disjoint interiors, the restriction of $f^{q}$ to any of these arcs is a bimodal map which fixes the end points, all these maps are smoothly conjugate to each other, and the set of end points of the arcs is the periodic orbit $\mathbf{O}_{\frac{P}{Q}}$.

Proof of Theorem 9. This proof is a repetition of the one given in [Boyl] and [MaT1] for some families of smooth maps. In the case when $q=1$, the Theorem follows directly from Theorem 7, hence we assume $q>1$ in the remainder of this proof. The orbit $\mathbf{O}_{\frac{P}{Q}}$ determines a partition in $q$ arcs of its complement in the circle. Let $J_{0}, J_{1}, \ldots, J_{q-1}$ be the corresponding closed segments, and $f_{i}$ (respectively $\left(f^{q}\right)_{i}$ ) be the restriction of $f$ (respectively $f^{q}$ ) to $J_{i}$. We assume $J_{0}$ is bounded by $P_{j}$ and $P_{k}$, where we use the notations of the paragraph before Theorem 7 . With these notations, $f_{0}$ has exactly two critical points (or turning intervals for plateau maps), while the other $f_{j}$ 's are linear maps. We have

$$
\begin{gathered}
\left(f^{q}\right)_{0}=f_{q-1} \circ f_{q-2} \circ \ldots \circ f_{0}, \\
\left(f^{q}\right)_{1}=f_{0} \circ f_{q-1} \circ \ldots \circ f_{1},
\end{gathered}
$$




$$
\left(f^{q}\right)_{q-1}=f_{q-2} \circ f_{q-3} \circ \ldots \circ f_{q-1}
$$

This implies that all these maps are bimodal and fix the end points. The conjugacy property comes from

$$
\begin{gathered}
\left(f^{q}\right)_{q-1}=\left(f_{q-1}\right)^{-1} \circ\left(f^{q}\right)_{0} \circ f_{q-1}, \\
\left(f^{q}\right)_{q-2}=\left(f_{q-2}\right)^{-1} \circ\left(f^{q}\right)_{q-1} \circ f_{q-2}, \\
\ldots \ldots \ldots \ldots \ldots \ldots \ldots \ldots \ldots \ldots \ldots \ldots \\
\left(f^{q}\right)_{1}=\left(f_{1}\right)^{-1} \circ\left(f^{q}\right)_{2} \circ f_{1},
\end{gathered}
$$

since $f_{1}, f_{2}, \ldots, f_{q-1}$ are homeomorphisms.

(Q.E.D. Theorem 9.)

\section{Topology and geometry of the boundaries of chaos and rotational chaos.}

Let $h(f)$ be the topological entropy $A_{-4}$ of $f$, and $F$ be some lift of $f$.

Consideration of the various definitions and results in Appendix A allows one to conclude that if $I(F)$ is not a single point, then $h(f)>0$. Explicitly, as we now explain, $f$ then has a horseshoe $_{A-4}$ and hence has positive topological entropy by Theorem D (see Appendix A-4).

Since by hypothesis $I(F)$ is not a single point, it contains two distinct numbers, say $\omega$ and $\theta$. By Corollary B' in Appendix A-2, one can find $x$ with rotation number $\omega$ and $y$ with rotation number $\theta$. Let $J$ stand for any of the two arcs bounded by the projection $X$ of $x$ and the projection $Y$ of $y$ : a high iterate of this arc covers the circle, hence $J$, as many times as we like. We shall say that a map $f$ has rotational chaos if $I(F)$ is not a single point for some lift $F$ of $f$, and is topologically chaotic (in short chaotic) if $h(f)>0$. Hence, by our remarks above, we see that rotational chaos implies chaos. However, the converse is not true, as some of the following results will explain. At this stage, we want to recall that, to the contrary of a claim often published in the physics literature, chaos, in any reasonable sense (not necessarily defined as we do by the positiveness of the topological entropy) is not ubiquitous as soon as one crosses the critical line: there are chaotic maps 
arbitrarily close to the critical line, but not arbitrarily close to all points on the critical line.

For the next few theorems, we need to introduce some additional definitions. Let $A_{p / q}^{s t a b}$ be that part of the region $A_{p / q}$ in which $f$ has a stable $q$-periodic point, with similar notations for $L_{p / q}^{s t a b}$, and let $L_{p / q}^{d b l}$ be that part of $L_{p / q}$ in which $f$ has only periodic orbits with periods of the form $2^{m} \cdot q$, for some $m \geq 0$.

Theorem 10: Region of zero topological entropy.

i) In any family $\mathbf{F}_{\delta}, h(f)=0 \quad \Leftrightarrow \quad f \in \bigcup_{\omega} L_{\omega}^{\ominus} \cup \bigcup_{p / q} L_{p / q}^{d b l}$

ii) Furthermore, in $\mathbf{F}_{t ; \delta}, h(f)=0 \Leftrightarrow f \in \bigcup_{\omega} L_{\omega}^{\ominus} \cup \bigcup_{p / q} L_{p / q}^{s t a b}$.

Proof of Theorem 10. Statement i) follows from the combination of Theorem 7 and Theorem C in Appendix A-4. Statement ii) is by combining i) with Theorem 16.

(Q.E.D. Theorem 10.)

Theorem 11: Topology and Geometry of the boundary of the circularly regular region. The boundary of the circularly regular region $\mathbf{F}^{\mathbf{c}-\mathbf{r e g}}$ of $\mathbf{F}_{\delta}$ defined by $\mathbf{F}^{\mathbf{c}-\mathbf{r e g}}=$ $\bigcup_{\omega \in \mathbb{R}} L_{\omega}$, is connected and locally connected, and has finite length.

Remark. The corresponding statements are known to be false in the standard family [BJa, FT].

Proof of Theorem 11. In both cases, from Theorem 2, we only need to prove that the height of $L_{p / q}^{+}$above the critical line is a function of $\frac{p}{q}$, with an upper bound going to 0 exponentially as $q \rightarrow \infty$. Slightly more than that is given by Lemma 11'.

Lemma 11': Height of $L_{p / q}^{+}$in $F_{\delta}$. The height of $L_{p / q}^{+}$above the critical line in $F_{\delta}$, is a decreasing function of $q$, with an upper bound going to 0 exponentially as $q \rightarrow \infty$.

(Q.E.D. Theorem 11.)

Proof of Lemma 11'. This amount to computations, since, by Theorems 7 and 9, we know that (using the notations of Theorem 9), at the upper tip of $L_{p / q}^{+},\left(f^{q}\right)_{i}$ is a map on 
an interval which covers the interior of this interval three times, and following the proof of Theorem 9 tells us more precisely what is this map.

We first treat the case of $F_{t ; \delta}$. Then, the map $\left(f^{q}\right)_{i}$ is as represented in Figure 6-a, i.e., is bimodal and fixes the end points, with two increasing segments of slope $S^{q}$, and one decreasing segment with slope $S^{q-1} \sigma$, where $\sigma=|s|=\frac{S(1-\delta)-1}{\delta}$. Since each of the segments where the map $\left(f^{q}\right)_{i}$ is monotone covers $J_{i}$, we have

$$
\left(\left|J_{i}\right|-\frac{2\left|J_{i}\right|}{S^{q}}\right) S^{q-1} \sigma=\left|J_{i}\right|,
$$

hence

$$
(1-\delta) S^{q+1}-S^{q}+(\delta-2) S+2=0 .
$$

It is easy to prove that $\left(^{*}\right)$ has a unique solution greater than $S_{c}=\frac{1}{1-\delta}$, which yields an alternate proof of Corollary 8'. What we seek here is an upper bound for this solution.

So let

$$
\begin{gathered}
g(x)=(1-\delta) x^{q+1}-x^{q}+(\delta-2) x+2, \\
g^{\prime}(x)=(q+1)(1-\delta) x^{q}-q x^{q-1}+(\delta-2), \\
g^{\prime \prime}(x)=q x^{q-2}(x(q+1)(1-\delta)+1-q) .
\end{gathered}
$$

Notice that $g^{\prime \prime}>0$ when $x \geq S_{c}$, that $g\left(S_{c}\right)=\frac{-\delta}{1-\delta}<0$, and that $g^{\prime}\left(S_{c}\right)=\frac{1}{(1-\delta)^{q}}+\delta-2$, hence $g^{\prime}\left(S_{c}\right)>0$ for $q$ large enough. One step of Newton's method with $S_{c}$ as a starting point yields

$$
y_{1}=\frac{1}{1-\delta}-\frac{\frac{-\delta}{1-\delta}}{\frac{1+(\delta-2)(1-\delta)^{q-1}}{(1-\delta)^{q-1}}},
$$

which, since $g^{\prime \prime}>0$, is an upper bound of the solution of $\left(^{*}\right)$ greater than $S_{c}$, and we have

$$
y_{1}-S_{c}=\frac{(1-\delta)^{q}}{(1-\delta)+(\delta-2)(1-\delta)^{q}}<2(1-\delta)^{q-1} .
$$

In the case of $F_{p ; \delta}$, the map $\left(f^{q}\right)_{i}$ is as represented in Figure 6-b, from which we get the height $h$ of $L_{p / q}^{+}$above the critical line:

$$
h=\frac{S_{c}-1}{2\left(S_{c}^{q}-1\right)},
$$


hence

$$
h<\frac{(1-\delta)^{q-1}}{2}
$$

This takes care of the estimates. The computation also shows that the height of $L_{p / q}^{+}$, in both families, only depends on $q$, hence is the same as the height of $L_{1 / q}^{+}$. By [BT], this implies that the height of $L_{p / q}^{+}$is a decreasing function of $q$.

(Q.E.D. Lemma 11'.)

The circularly regular region for the tip maps with $\delta=\frac{1}{4}$ is illustrated in Figure 7. Only the contributions of a few $L_{p / q}$ 's $\left(\frac{p}{q} \in\left[0, \frac{1}{2}\right]\right.$ with $\left.q \leq 11\right)$ are represented.

\section{Theorem 12: Topology and Geometry of the boundary of the topologically} regular region. The boundary of the topologically regular region $\mathbf{F}^{\mathbf{r e g}}$ of $\mathbf{F}_{\delta}$ defined by $f \in \mathbf{F}^{\mathrm{reg}} \Leftrightarrow h(f)=0$, is connected and locally connected and has finite length, except in the plateau case when $S$ is too small, in which case, it is made of arcs of finite length with both end points on the upper boundary $H=H_{\max }$ of the parameter space.

Proof of Theorem 12. We first consider the case of tips maps. By Theorem 10, we have to control the boundary of $L_{p / q}^{+, s t a b}$ in $L_{p / q}$. This boundary is where $\sigma S^{q-1}=1$, hence $S$ is the greatest root of $(1-\delta) x^{q}-x^{q-1}-\delta=0$ (hence is a strictly decreasing function of $\mathrm{q}$ ), and the boundary of $L_{p / q}^{+, s t a b}$ in $L_{p / q}$ is a segment parallel to the $\omega$ axis. Since it is clear (from the proof of Theorem 11) that the tip of $L_{p / q}$ is beyond the boundary of $L_{p / q}^{+, s t a b}$, the estimate in the proof of Theorem 11 implies the tip maps part of Theorem 12.

The case of the plateau maps is solved by the following Lemma, together with the estimate in the proof of Theorem 12, since the tip of $L_{p / q}$ corresponds to a chaotic map.

\section{Lemma 12': The boundary of topological chaos in $L_{p / q}$ for plateau maps.}

- When going up from the critical line to the positive entropy region inside $L_{p / q}$ in $\mathbf{F}_{p ; \delta}$, one must cross a complete cascade of period doubling bifurcations.

- The boundary of the region where $h\left(p_{\Omega, U ; \delta}\right)=0$ in $L_{p / q}^{+}$is a connected and locally connected curve, made of at most countably many smooth pieces crossing the gaps of a Cantor set imbedded in the two dimensional parameter space. 
- This boundary has finite length, bounded by $\frac{1}{\delta}$ times the width of $L_{p / q}$ on the critical line.

- Maps corresponding to parameter values on this curve have zero topological entropy

(Q.E.D. Theorem 12.)

Proof of Lemma 12'. In Appendix B, we show that, for each $S>1$, the parameter space for the maps $c_{S, \mu ; a, b}$ is a 3 -cell $\mathbf{C}_{S}$. Now remark that for any plateau map $f=p_{\Omega, U ; \delta}$ in $L_{p / q}^{+}$, where $U=S+H$, the $q^{\text {th }}$ iterate of $f$ restricted to any $I_{i}$ and renormalized to the unit interval, is some map $c_{S^{q}, \mu ; a, b}$. From the proof of Theorem 2, we know that all $L_{p / q}^{+}$'s are exact copies of each other, up to uniform dilatation, and a computation shows that, up to a homothetie, $L_{p / q}^{+}$is the surface $\Pi_{S^{q}}$ in $\mathbf{C}_{S^{q}}$, as represented in Figure 8 according to whether $S^{q} \leq 3,3<S^{q}<5$, or $5 \leq S^{q}$. It is easy to check that horizontal lines in the $(\Omega, S)$-plane are sent to horizontal lines in the $(a, b)$-plane, and that vertical lines in the $(\Omega, S)$-plane are sent to vertical lines in the $(a, b)$-plane, hence $\Pi_{S^{q}}$ is a plane, and cuts $\mathbf{C}_{S^{q}}$ on a triangle $\mathbf{T}_{S^{q}}$ symmetrical with respect to its height: we leave to the reader to check that the equation of this plane reads $\mu=\frac{1}{2 S^{q}}\left[S^{q}+1+2(a-b)\right]$.

The boundary of chaos in each $\mu$-cut of $\mathbf{C}_{S^{q}}$, is described by Theorem E, and consists in the closure of a set of lines with slopes 1 or -1 . By Theorem $F$, the surface corresponding to one of these lines as $\mu$ varies cuts $\Pi_{S^{q}}$ in a line whose absolute value of the slope at each point is not greater than the slope of the sides of the triangle. The homothetie of the triangle $\mathbf{T}_{S^{q}}$ to $L_{p / q}^{+}$gives it sides with $\frac{1}{\delta}$ as absolute value of the slope.

(Q.E.D. Lemma 12'.)

The region of zero topological entropy for the tip maps with $\delta=\frac{1}{4}$ is illustrated in Figure 9. Only the contributions of a few $L_{p / q}^{s t a b}$ 's $\left(\frac{p}{q} \in\left[0, \frac{1}{2}\right]\right.$ with $\left.q \leq 11\right)$ are represented.

Remarks. As for Theorem 11, the statements corresponding to Theorem 12 are known to be false in the standard family $[\mathrm{FT}]$. We conjecture that the boundary of chaos inside each the tongues of the standard family is of finite length.

Figure 10 displays some bifurcation lines of the plateau family with $\delta=\frac{1}{2}$. The second remark after the proof of Lemma F' in Appendix B explains some of the straight 
lines one observes in this figure, at least the piece of these straight lines insides the $L_{p / q}^{+}$'s. To understand these straight lines more globally, notice that the condition for a plateau boundary to be part of a periodic orbit with a given combinatorics corresponds to a linear relation between $H$ and $\Omega$ : this is because of the way $H$ and $\Omega$ appear in the formula of the maps. The $S-H$ or $S-\Omega$ relations would be more complicated.

Theorem 13: A stratification of the plateau family. For each $c>0$, the region of the parameter space of $\mathbf{F}_{p ; \delta}$ where $h(f)=c$, is connected and simply connected, except when $S$ is too small, in which case, each connected component contains two pieces of the upper boundary $H=H_{\max }$ of the parameter space. When $U$ is increased above the critical line in $p_{\Omega, U ; \delta}$ (with $\Omega$ and $\delta$ kept fixed), no periodic orbit is destroyed.

Proof of Theorem 13. The second statement is an easy consequence of the fact that varying $U$ above the critical line in $p_{\Omega, U ; \delta}$ with $\Omega$ and $\delta$ kept fixed, correspond to a stunted family. Similarly, the topological entropy is a non-decreasing and continuous function of $U$, with $\Omega$ and $\delta$ kept fixed. Since the entropy is continuous in $\mathbf{F}_{p ; \delta}$, the first statement follows (see $[\mathrm{Msz}])$.

(Q.E.D. Theorem 13.)

\section{Supercritical tip maps with stable behavior.}

We begin with a completely elementary (i.e., both the techniques and concepts are elementary) proof of the following result, which can also be obtained along the lines of the proof of Theorem 16 .

Theorem 14: Stability implies Well-Orderedness for periodic orbits. Any stable periodic orbit of a tip map is $\frac{p}{q}$ - ordered for some $\frac{p}{q}$. Any marginally stable periodic orbit $\mathbf{O}$ of a tip map $f$ is either $\frac{p}{q}$-ordered for some $\frac{p}{q}$, or $f$ has a marginally stable periodic orbit $\mathbf{O}^{\prime}$ which is $\frac{p}{q}$-ordered for some $\frac{p}{q}$, the product of the slopes along the orbits is -1 , and $\mathbf{O}^{\prime}$ has period $2 q$ and rotation number $\frac{p}{q}$.

Proof of Theorem 14. Only maps above the critical line need to be considered, since the statement is obvious elsewhere (where only the first case occurs in the marginally stable 
case). In this proof $[\alpha, \beta]$ stands for the arc delimited by $\alpha$ and $\beta$, where the orientation is not specified as going counterclockwise from $\alpha$ to $\beta$, but by the fact that $[\alpha, \beta] \subset I_{\delta}$, where $I_{\delta}$ is the arc going counterclockwise from $\frac{1-\delta}{2}$ to $\frac{1+\delta}{2}$.

- We first consider the stable case.

Hence, assume some supercritical tip map $f$ has a stable periodic orbit $\mathbf{O}$ with period $q$, which is not $\frac{p}{q}$-ordered for any $p$. We shall organize the proof by the number $m$ of points of $\mathbf{O}$ belonging to the interior of the $\operatorname{arc} I_{\delta}$ (which is the arc carrying the slope $s$ ). Notice that the absolute value of the slope of $f^{q}$ at any point of $\mathbf{O}$ is given by $\sigma^{m} S^{q-m}$, where we set again $\sigma=|s|$, and stability means $\sigma^{m} S^{q-m}<1$.

- Since $S>1$ above the critical line, the case $m=0$ is solved trivially.

- The case $m=1$ can be reduced to the case $m>1$ by a simple surgery method as follows. Let $x$ be the unique point of $\mathbf{O}$ in $I_{\delta}, x_{L}$ and $x_{R}$ the neighbors of $x$ in $\mathbf{O}$, and $X, X_{L}$ and $X_{R}$ lifts of these points which are consecutive among lifts of point in $\mathbf{O}$. Since $\mathbf{O}$ is not $\frac{p^{\prime}}{q^{\prime}}$ - ordered for any $\frac{p^{\prime}}{q^{\prime}}$, either $F\left(X_{L}\right)>F(X)$ or $F\left(X_{R}\right)<F(X)$ for any lift $F$ of $f$. It is then easy to construct a circle map $f^{\prime}$ such that $\mathbf{O}$ is an orbit of $f^{\prime}$, and the method described below for $m \geq 2$ works for $f^{\prime}$ : the details are explained in Figure 11 in the case $F\left(X_{L}\right)>F(X)$.

- In the case $m>1$, let $a_{1}, a_{2}, \ldots, a_{m}$ be the points of $\mathbf{O}$ in $I_{\delta}$, ordered clockwise. Define $n(i)$ as the time the orbit of $a_{i}$ spends out of $I_{\delta}$ before returning to $I_{\delta}$, i.e.,

$$
f^{n(i)}\left(a_{i}\right) \in \operatorname{Interior}\left(I_{\delta}\right), \text { and } f^{m}\left(a_{i}\right) \notin \operatorname{Interior}\left(I_{\delta}\right) \text { for } 0<m<n(i)
$$

and notice that, for $n=\inf _{i}(n(i))$, we have $\sigma S^{n}<1$.

Let $j$ stand for the smallest $i$ such that $n(i)=n$.

- If $j=1$, with $a_{k}=f^{n}\left(a_{1}\right)$, the image under $f^{n}$ of the arc $\left[a_{1}, a_{k}\right]$ has to be strictly included in itself by $\sigma S^{n}<1$, but this is impossible because there are exactly $k$ points of $\mathbf{O}$ in this arc.

- The case $j=m$ is treated as the case $j=1$.

- If $j \notin\{1, m\}$, set $a_{k}=f\left(a_{j}\right)$.

- If $k=1$, it is plain that $f^{n}\left(a_{j-1}\right) \in I_{\delta}$, which contradicts the minimality of $j$.

- If $k=m$, argue like when $j=1$. 
- If $k \notin\{1, m\}, f^{n}\left(a_{j-1}\right)$ has to be out of $I_{\delta}$, otherwise, argue like when $k=1$. Since $\sigma S^{n}<1$, the arc $\left[a_{j-1}, a_{j}\right]$ has to be longer than the arc $\left[a_{j}, a_{m}\right]$, which, by the same $\sigma S^{n}<1$, implies that $f^{n}\left(\left[a_{j}, a_{m}\right]\right)$ has to be included in $\left[a_{j-1}, a_{j}\right]$, but this is not possible since there is no point of $\mathbf{O}$ in this arc.

- We now consider the marginally stable case, i.e., $\sigma^{m} S^{q-m}=1$. If $n(i)$ is not constant, we proceed as before. So assume that $n(i)=n$ for all $n$, and that $\mathbf{O}$ has period $q$, but is not $\frac{p}{q}$ - ordered for any $p$. Then, $\sigma S^{n}=1$, so that if $f^{n}\left(A_{s}\right)=A_{t}$, we also have $f^{n}\left(A_{t}\right)=A_{s}$, and $m=2$. The middle point of the arc $\left[A_{s}, A_{t}\right]$ has to be periodic with period $\frac{q}{2}$, and if it would not be $\frac{p^{\prime}}{\frac{q}{2}}$-ordered, we would get a contradiction by modifying $f$ so that the orbit of this point is preserved, but the orbit is stable.

(Q.E.D. Theorem 14.)

The following result is not only completely elementary, but also quite easy, the only tricky case being covered in the discussion for Theorem 4. The proof is left to the reader, with the special case of a stable fixed point explained in Figure 12.

Theorem 15: A corner bounds each basin. Given a tip or plateau map $f$, for each stable periodic orbit with period $p$, there is a point $x$ in the orbit such that the shortest of the intervals centered at $x$ and bounded by one of the corners $\frac{1-\delta}{2}$ and $\frac{1+\delta}{2}$, is mapped into itself by $f^{p}$. Hence one of the corners is in the immediate basin of attraction of any stable periodic orbit, and there are at most two stable periodic orbits. If $f$ is not supercritical, both corners are in the immediate basin of attraction of any stable periodic orbit, but generally not in the same connected component of the basin. Hence there is at most one stable periodic orbit. If stable is replaced by marginally stable, and the slope is one, $f^{q}$ is a rotation. If stable is replaced by marginally stable, and the slope is -1 , there is a periodic interval, and all points in it, except for the original periodic orbit, have period $2 q$.

Remark. It follows readily from Theorem 15 that there are at most 2 stable periodic orbits for any map in the family, a statement also known to be true for the standard family, and easy to prove in the plateau case. The general organization of the stability and bistability regions in the parameter space of the standard family is closer to what we get 
for our plateau maps (conjecturally, the differences between the two cases are metric, but not topological except for the existence of behavior in the plateau case not matched in the standard family inside the $L_{p / q}$ 's, and what is covered by Theorems 11 and 12), than to the peculiar structure in the tip cases, to be described in Theorem 17. In the subcritical case, bounds on the number of periodic orbits for real analytic maps beyond the case of the standard family are given in [He3].

Theorem 16: When there is no stable nicely ordered periodic orbit, some iterate has all slopes out of $[\mathbf{- 1 , 1}]$. Let $f$ be a supercritical tip map with no stable or marginally stable, nicely ordered periodic orbit. Then for some $n$, the absolute value of the slopes of $f^{n}$ are all greater than one.

Remark. Theorems 14 and 16 can be reinterpreted as giving new characterizations of nice order, a question which has been the object of many investigations since the $18^{\text {th }}$ century (see [Be], [Ch], [Sm], [Ma], [MH], as well as [STZ] and references therein).

Proof of Theorem 16. Assume Theorem 16 is false. Then there exists some $x \in I_{\delta}$ and a smallest $n>0$ such that $f^{n+1}(x) \in I_{\delta}$. It is then clear that $s S^{n} \leq 1$. Let $I_{x}$ be the maximal interval containing $x$ and such that $f^{n+1}(y) \in I_{\delta}$ for all $y \in I_{x}$. Since $f^{n+1}$ is orientation reversing and either contacting or isometric on $I_{x}$, it is easy to deduce that $I_{x}$ must contain an end point of $I_{\delta}$ and to further deduce that $I_{x}$ must contain a fixed point of $f^{n+1}$, hence a stable periodic orbit for $f$. The rest follows from Theorem 14 .

(Q.E.D. Theorem 16.)

Remark. The proof given here of Theorem 16 is quite specific to the tip maps, which makes this theorem quite remarkable: one would more generally like to know whether a piecewise linear map which is not a homeomorphism and which does not have a stable periodic orbit must have an iterate with all slopes greater than one in absolute value. In this context see the results and the discussion in [MTr].

Corollary 16': Density of the preimages of the turning points when no stable nicely ordered orbit exists. For any tip map with no stable nicely ordered periodic orbit, the set of all preimages of the corners $\frac{1-\delta}{2}$ and $\frac{1+\delta}{2}$ is dense in the circle. 
For the next theorems, we define the stability region $\mathbf{R}_{\delta}$ of $\mathbf{F}_{t ; \delta}$ as the set of $t_{\Omega, S ; \delta}$ 's with $0 \leq \Omega<1$ in $\bigcup_{p / q}$ interior $\left(A_{p / q}^{\oplus, s t a b}\right)$. The way the stability region is placed with respect to the $A_{p / q}$ 's is illustrated in Figure 13 for $\delta=\frac{1}{4}$, and global views of $\mathbf{R}_{\delta}$ are displayed in Figure 14 for $\delta=\frac{1}{2}$ and $\delta=\frac{3}{4}$. Until the end of the main text, fractions are supposed to belong to the interval $[0,1]$. The Farey sequence of order $i$ is the ordered set of reduced fractions in $[0,1]$, with denominators up to $i$. Two fractions $\frac{p}{q}$ and $\frac{p^{\prime}}{q^{\prime}}$ in $[0,1]$ are Farey neighbors if they are consecutive in some Farey sequence. Let $N(i)$ be the number of terms in the Farey sequence of order $i$ for $i>0$, with $N(0)=1$, and let $N^{\prime}(i)=N(i)-1$.

Theorem 17: Topology of the stability region, $\mathbf{R}_{\delta}$, for $\mathbf{F}_{t ; \delta}$. (First "Zipper Theorem") There exists a strictly decreasing sequence, $\delta_{i}$, with $\delta_{i}=1-2^{-1 / i}, i \geq 0$ such that for $\delta \in\left(\delta_{i+1}, \delta_{i}\right), \mathbf{R}_{\delta}$ has $N(i)+N^{\prime}(i)$ connected components, $N(i)$ of which are simply connected, the others being infinitely connected (i.e., possessing infinitely many holes). Furthermore, $A_{p / q}^{\oplus, s t a b}$ can intersect $A_{p^{\prime} / q^{\prime}}^{\oplus, s t a b}$, and does intersect it for $\delta$ big enough, if and only if $\frac{p}{q}$ and $\frac{p^{\prime}}{q^{\prime}}$ are Farey neighbors.

Proof of Theorem 17. The last statement follows trivially from the fact that the height of $L_{p / q}^{+, s t a b}$ is a strictly decreasing function of $q$ (see the proof of Theorem 12), and the definition of Farey neighbors. So let $\frac{p}{q}$ be some rational number in $[0,1]$, and $\frac{p(i)}{q(i)}$ be the sequence of its Farey neighbors on one side, with $q(i+1)>q(i)>q$ : we want to show that the value $\delta\left(\frac{p}{q}, \frac{p(i)}{q(i)}\right)$ of the parameter $\delta$, at which $A_{p / q}^{\oplus, s t a b}$ and $A_{p(i) / q(i)}^{\oplus, s t a b}$ detach from each other, decreases when $i$ increases (hence a zipper effect, except for the case when $q=1$ where the detachment value $\delta=.5$ does not depend on $i$ ), with the limit value $\delta_{q}$ a decreasing function of $q$.

Let us consider the case when $\frac{p(i)}{q(i)}>\frac{p}{q}$, the other case being treated in the same way. We consider a map $t_{\Omega, S ; \delta}$ at the intersection of the right boundary of $A_{p / q}^{\oplus, s t a b}$ with the left boundary of $A_{p(i) / q(i)}$, and need to express the fact that $S$ corresponds to the top of the stability region $A_{p(i) / q(i)}^{\oplus, s t a b}$. Let $T_{\omega, S ; \delta}$ be the lift of $t_{\Omega, S ; \delta}$ such that the lifts of $\frac{1+\delta}{2}$ are fixed points under $T_{\omega, S ; \delta}^{q}$ (this choice can be made because we work on the right boundary of $\left.A_{p / q}^{\oplus, s t a b}\right)$. Then the graph of $T_{\omega, S ; \delta}^{q}$ is a zigzag line creeping along the first 
diagonal; together with the first diagonal, this graph determines a sequence of $q$ triangles per interval of the form $I_{n}=\left[n+\frac{1+\delta}{2}, n+1+\frac{1+\delta}{2}\right)$. The right triangle in $I_{n}$ has $x$-width $\delta+a_{q}$, and the remaining ones are similar copies, with scaling factors $\frac{1}{S}$ to $\left(\frac{1}{S}\right)^{q-1}$, hence

$$
\left(\delta+a_{q}\right)\left[1+\frac{1}{S}+\ldots+\left(\frac{1}{S}\right)^{q-1}\right]=1 .
$$

Each of these triangles has slopes $S^{q}$ and $s S^{q-1}$, and since we are on the left boundary of the top of $A_{p(i) / q(i)}^{\oplus, s t a b}$, we have

$$
\sigma S^{q(i)-1}=1 .
$$

Each triangle is followed (to the right) by one which is either bigger or smaller, in the way the residue classes $\bmod q$ are organized: the description of the organization of residue classes $\bmod q$ was performed by E. B. Christoffel in the last century [Ch], and it is explained in [STZ] how this relates to the kneading theory of circle maps, as investigated (with a different name) one century before as a question in number theory by Johan Bernoulli (the astronomer of the famous family) [Be], and contemporarily by Marston Morse and Gustav Hedlund $[\mathrm{MH}]$ in the context of symbolic dynamics. On the other hand, because we are on the left boundary of $A_{p(i) / q(i)}$, the tips of the triangle lift a periodic orbit with period $q(i)$ : since $p(i) / q(i)$ and $\frac{p}{q}$ are Farey neighbors, we get from the kneading theory of degree two circle maps that there are either $n$ or $n+1$ points of the orbit of $\frac{1-\delta}{2}$ between two consecutive points of the orbit of $\frac{1+\delta}{2}$ (i.e., in a triangle) [GoT]. More precisely, there are $n$ points if the triangle is smaller than the previous one, and $n+1$ points otherwise. Putting together all this kneading information, basically easy but too long to be recalled here in details (see [STZ], [GoT] and references therein), the fact that the tip of one triangle is the $n^{\text {th }}$ or $(n+1)^{\text {th }}$ image of the previous tip yields

$$
\frac{a_{q}}{\delta}=\sigma S^{q(i)-1}
$$

Reassembling the equations gotten so far, we get

$$
\delta=\frac{1}{2} \cdot \frac{S^{q-1}}{1+S+\ldots+S^{q-1}} .
$$

This last expression increases with $S$, hence the zipper effect, except for $q=1$. The limit value $\delta_{q}$, corresponding to $S=S_{c}=\frac{1}{1-\delta}$, gives an explicit equation for $\delta_{q}$, to wit

$$
1 /(2 \delta)=1+(1-\delta)+(1-\delta)^{2}+\cdots+(1-\delta)^{q-1},
$$


hence

$$
\delta_{q}=1-2^{-1 / q}
$$

(Q.E.D. Theorem 17.)

The bifurcations at the first three $\delta_{i}$ 's are illustrated by Figure 15 .

A subset $A$ of the circle is a circularly ordered chaotic attractor for $f$, with rotation number $p / q$, if $A$ is a chaotic attractor and for some nicely ordered periodic orbit $P_{0}, \ldots, P_{q-1}$ with successive indices around the circle, and with rotation number $p / q$,

$$
x \in A \cap I_{i} \Rightarrow f(x) \in A \cap I_{(i+p)_{1}},
$$

where $I_{i}$ is the $\operatorname{arc}\left(P_{i}, P_{(i+1)_{1}}\right)$. An example of coexistence of stable periodic orbit and circularly ordered chaotic attractor is illustrated in Figure 16.

Let $\mathbf{R}_{\delta}^{\prime}$ be the set of tip maps $t_{\Omega, S ; \delta}$ that have a stable periodic orbit or a circularly ordered attractor.

Theorem 18: Topology of the $\mathbf{R}_{\delta}^{\prime}$ 's. (Second "Zipper Theorem") The statements of Theorem 17 apply to $\mathbf{R}_{\delta}^{\prime}$ with a sequence $\delta_{i}^{\prime}$, where $\delta_{i}^{\prime}>\delta_{i}$, and with $A_{p / q}^{\oplus, s t a b}$ extended to include the maps in $A_{p / q}^{\oplus}$ with circularly ordered chaotic attractors.

Proof of Theorem 18. The proof goes essentially as for the preceding theorem, the only difference being that the boundary of $A_{p(i) / q(i)}^{\oplus, s t a b}$ condition has to be replaced by a relation describing the boundary of the circularly ordered chaotic region with rotation number $\frac{p(i)}{q(i)}$. This equation is gotten by the geometry of the graph of the restriction $t_{\Omega, S ; \delta}^{q(i)}$ to a $J_{k}$ (with the notations of the proof of Theorem 9), as depicted in Figure 17, and reads

$$
\frac{1}{S^{q(i)}}+\frac{1}{\sigma S^{q(i)-1}}=1 .
$$

Combining this with the other relations yields

$$
S^{q(i)+1}(1-\delta)-S^{q(i)}-S+1=0,
$$

hence

$$
\delta=\frac{1}{2+\frac{1}{S^{q(i)-1}}} \cdot \frac{S^{q-1}}{1+S+\ldots+S^{q-1}}
$$


with the same limit value

$$
\delta_{q}^{\prime \prime}=1-2^{-1 / q}
$$

as before, but no control of the zipper effect yet, because of the $q(i)$ dependence in the formula for $\delta$. However, since $\delta$ decreases when $q$ increases, the following can be shown:

Let $R_{p / q}^{\prime}$ be the closure of the subset of $A_{p / q}^{\oplus}$ where the maps have stable periodic orbits or circularly ordered chaotic attractors. As $\delta$ decreases to $\delta_{q}^{\prime}$, the $R_{r / s}^{\prime}$ 's (where $\frac{r}{s}$ is a Farey neighbor of $\frac{p}{q}$ ) with bigger denominators detach themselves from $R_{p / q}^{\prime}$ before those with smaller denominators do. That is, the unzipping takes place in the reverse order from that in Theorem 17.

(Q.E.D. Theorem 18.)

The region $\mathbf{R}_{\delta}^{\prime}$ is shown in Figure 18 for $\delta=\delta_{3}^{\prime}$.

\section{Summary, Discussion, and Open Issues}

Since it is virtually impossible to summarize the results of eighteen theorems and many observations in a concise manner in words, we will limit our "summary" to the sketch in Figure 19, which illustrates many of the phenomena we have described by displaying the structure of parameter space for the tip maps with $\delta>\frac{1}{2}$ around $A_{1 / 2}$. We will turn in the rest of this section to a discussion of the implications of our results and of some important remaining open issues.

Apart from their interest as dynamical systems in their own right, the piecewise linear circle maps have proven to be interesting and valuable in several other contexts. First, as a pedagogical example of a non-trivial, essentially solvable model for mode locking and the quasi-periodic transition to chaos, one can use them to clarify with explicit examples and calculations concepts that require much deeper analysis in the general smooth case. Second, our analytic insight into piecewise linear maps has both stimulated conjectures and suggested methods of proof that apply to the general smooth case. Specifically, after a lecture given on this material, a number of conjectures on the smooth case were indeed proved [EKT] by Adam Epstein, Linda Keen and one of the authors (C.T.), using a method initiated by Bill Thurston in the context of interval maps. The [EKT] results are parallel to (but slightly less strong) than those we obtained in $\S 8$ above in a mostly trivial manner 
for the plateau map families. For the tip family, the counterpart to the results in [EKT] is reasonably easy, but to go beyond, we needed a much deeper analysis which was carried out by Marco Martens with two of us (R.G. and C.T.), as reported in [GMT]. In any case, the piecewise linear maps have already served well in this second role. Third, although in view of the seeming non-generic nature of the non-smooth map, one might expect that finding a physical realization of phenomena such as the "Arnold sausages" would be difficult; in fact, again following a lecture on this material, preliminary numerical simulations at the University of Frankfurt [ZKM] suggest that the phenomena should be experimentally observable in driven Josephson junctions; further work on this is in progress.

Among the other open issues, perhaps the chief matter left unresolved by our study is the measure of the mode-locked intervals below the critical line. Although our numerical data strongly suggest that this measure is strictly less than one for all $\delta<1$ and for all $S<S_{c}$, we have at present no proof of this conjecture.

A second matter worth further study is motivated by adopting the numerical analyst's perspective and considering the piecewise linear maps as "finite element, linear spline approximations" to the smooth case. This view suggests the study of "higher-order" piecewise linear circle maps - i.e., multi-spline fits having more than two independent slopes - and, in principle, of studying the convergence properties of the bifurcation sequences of these maps to those of the smooth case as the number of splines increases. Indeed, an analogous study $[\mathrm{HC}]$ comparing the (two-spline) tent and (three-spline) trapezoidal maps to the (four-spline) house map shows that, as expected, the house map captures more of the bifurcation sequence of the logistic map than its simpler cousins. However, the effort required to undertake detailed studies of the multiple-parameter, higher-order piecewise linear maps suggests that one should have a clear understanding of the importance of the questions being studied before proceeding. Further, a recent study [MT] has shown rigorously that no finite segment PWL map with no zero slopes can capture the full renormalization sequence of the logistic map.

\section{Acknowledgements}

Over the long course of this work we have benefited from interactions with a large 
number of colleagues. A major mathematical motivation for this project was the resistance to proof of some conjectures about the standard family of circle maps. As noted above, through discussions with Adam Epstein, Linda Keen, and Marco Martens, we not only gained insight into the present work (as reflected in [GMT]) but were also able to prove some of these conjectures in $[\mathrm{EKT}]$. It is thus a great pleasure to acknowledge the value of our discussions with those colleagues, as well as with Roy Adler, Kostya Khanin, Bruce Kitchens, Thomas Novicky and Michael Shub, whose patient listening helped us find and/or fix proofs for numerous statements reported here. We also enjoyed many valuable discussions about the phenomenon of mode locking in the physical world with Leon Glass, Michael Mackey, Ronnie Maineri, and Steve Strogatz. We are grateful to Werner Martienssen, Uwe Krüger, and Holger Zimmerman for sharing with us their preliminary observation of "Arnold sausages" in a model of a driven Josephson junction. 


\section{Appendix A: Essential Definitions and Background Material.}

This Appendix assembles some background material on the dynamics of circle maps, interval maps, and topological entropy: its only use for experts is to establish the notation we use in the rest of the paper. Non-experts are advised to sketch figures, wherever appropriate, of each of the definitions, concepts, and arguments, as this will greatly improve their understanding of the paper. As in the main text, all fractions will be written in lowest order terms, except where otherwise specified, and we shall use the notation $(z)_{n} \stackrel{\text { def }}{=}$ $z \bmod n$.

\section{A-1. Circle maps, lifts, and degree.}

When dealing with the dynamics of circle mappings, it is useful to consider the circle as the set of real numbers $\bmod 1$, i.e., to think of the circle as being the set $\mathbb{T}=\mathbb{R} / \mathbb{Z}$, meaning that we consider two real numbers $x, y$ in the set of real numbers $\mathbb{R}$ to be equivalent if and only if they differ by some integer $n$ in the set $\mathbb{Z}$ of rational integers. This means that our angles are measured in the unit $2 \pi$ radians.

The circle $\mathbb{T}$ is then the set of the equivalence classes so defined, a good set of representatives of these equivalence classes being the semi-open interval $[0,1)$. The topology of the circle is obtained by endowing $\mathbb{T}$ with the distance $d$ defined by:

$$
d\left((x)_{1},(y)_{1}\right)=\min \left(\left|(x)_{1}-(y)_{1}\right|,\left|(x)_{1}\right|+\left|1-(y)_{1}\right|,\left|(y)_{1}\right|+\left|1-(x)_{1}\right|\right) .
$$

To each continuous circle map $f: \mathbb{T} \rightarrow \mathbb{T}$, we can associate lift maps, where the continuous map $F: \mathbb{R} \rightarrow \mathbb{R}$ is a lift of $f$ if and only if

$$
(F(x))_{1}=f\left((x)_{1}\right) .
$$

There is a countable infinity of such lifts for any $f$ : if $F_{0}$ is one of the lifts of $f$, the set of lifts of $f$ is the set of maps $F_{n}=F_{0}+n$, where $n \in \mathbb{Z}$. On some occasions, and in particular for the purpose of drawing pictures, it is easier to suppose that $F_{0}(0)$ belongs to the interval $[0,1)$. However, this convention cannot hold when we interpolate the successive lifts $F_{n}$ of some maps $f$, by a continuous one parameter family $\left\{F_{\mu}\right\}$ with $F_{(\mu+1)}=F_{\mu}+1$. 
If $F$ is the lift of a continuous circle map $f$, there exists an integer $d$ such that

$$
F(x+1)=F(x)+d
$$

for all real numbers $x$. This number $d$ is called the degree of $f$ (or of $F$ ), and a good intuition of its meaning can be gotten by contemplating the graphs for maps with degrees respectively equal to $-1,0,1$ and 2 in Figure A1: $d$ is the algebraic number of times the circle is wrapped onto itself by the map.

The rotations $R_{\theta}(x)=(x+\theta)_{1}$ have degree one. Since the degree varies continuously for continuous deformations of the maps, and since we are interested in parametrized continuous families containing the rotations, we only consider degree one in the paper and the rest of the Appendix. Hence, throughout the main text and the rest of the appendices circle map will always mean degree-one continuous circle map, and a map will be called a lift if and only if it is the lift of a degree one circle map.

\section{A-2. Rotation intervals and rotation numbers.}

Let $f$ be a circle map, and $F$ be a lift of $f$. Using the notation $F^{n}(x)$ for the $n^{t h}$ iterate of $F$, we set

$$
\begin{aligned}
& \underline{\rho}_{F}(x)=\liminf _{n \rightarrow \infty} \frac{F^{n}(x)}{n}, \\
& \bar{\rho}_{F}(x)=\limsup _{n \rightarrow \infty} \frac{F^{n}(x)}{n},
\end{aligned}
$$

and define the rotation interval of $F[\mathrm{NPT}]$ as

$$
I(F)=[a, b]
$$

where

$$
a=\inf _{x \in \mathbb{R}} \underline{\rho}_{F}(x), b=\sup _{x \in \mathbb{R}} \bar{\rho}_{F}(x) .
$$

In some cases, and in particular when $F$ is non-decreasing, $I(F)$ reduces to a single number, also denoted $\rho(F)$, called the rotation number of $F[\mathrm{Po}]$; then the limsup and liminf can be replaced by a limit in the defining formulas. In this case, $(\rho(F))_{1}$ is called the rotation number of $f$. Similar passing from $F$ to the circle map $f$ itself is in general 
impractical because the rotation interval of a general lift may be longer than one. Intuitively, the uniqueness of the rotation number when $F$ is non-decreasing can be pictured by what happens when cars race on a circuit where the road is too narrow to allow passing.

When $I(F)$ is a single point, $f$ has a periodic orbit if and only if $\rho(F)$ is a rational number. Furthermore, if $F$ is non-decreasing with $\rho(F)=\frac{p}{q}$, the period of all periodic orbits of $f$ is equal to $q$. This number $q$ is not alone sufficient to allow a combinatorial description of the dynamics of $f$ restricted to these periodic orbits: with $\frac{P}{Q}=\left(\frac{p}{q}\right)_{1}, P-1$ is the number of points of the orbit are jumped over from a point to its image, in the positive direction around the circle (equivalently, $P$ is the number of revolutions around the circle which is needed to come back to any point when following the orbit). Thus this number is constant on the points belonging to all periodic orbits of $f$ when $F$ is non-decreasing with rational rotation number $\frac{p}{q}$. In other words, the dynamics of such a map $f$, restricted to any of its periodic orbits, is combinatorially the same as the dynamics of the rotation by $\frac{P}{Q}$. The number $p$ is also the number of orbits of $F$ (forward and backward) which project to any given periodic orbit of $f$ by $\bmod 1$.

Non-decreasing maps form an important class in the study of degree-one endomorphisms. Following Alain Chenciner, we designate as fomeomorphisms (a French contraction for "false homeomorphism") the continuous degree-one circle maps whose lifts are non-decreasing. A periodic orbit of a circle map, which is also a periodic orbit of some fomeomorphism with rotation number $\frac{P}{Q}$ (hence combinatorially like an orbit of the rotation by the angle $\frac{P}{Q}$ ) is called $\frac{P}{Q}$-ordered. More generally, an orbit of a circle map, which is also an orbit of some fomeomorphism with rotation number $\Omega$, is called $\Omega$-ordered. Lifts of such orbits are then said to be $\omega$-ordered for some $\omega$ such that $\Omega=(\omega)_{1}$. In particular, a $\frac{p}{q}$-ordered orbit is always the lift of some periodic orbit, invariant under a fomeomorphism with rotation number $\frac{P}{Q}=\left(\frac{p}{q}\right)_{1}$. We sometimes say nicely ordered for " $\omega$-ordered for some $\omega^{\prime \prime}$.

We denote by $\mathcal{F}^{0}(\mathbb{R})$ the space of lifts of fomeomorphisms equipped with the sup norm: i.e., two such maps are close together if their respective values at each point of an interval of length one are close to each other. More generally, $\mathcal{F}^{k}(\mathbb{R})$ stands for the space of $k$ times continuously differentiable lifts of fomeomorphisms, where two of them are close 
together if the maps and their $k$ first derivatives are. Further, we shall use the following definitions for inequalities between real maps:

$$
F>G \Leftrightarrow \forall x, F(x)>G(x),
$$

and

$$
F \geq G \Leftrightarrow \forall x, F(x) \geq G(x) .
$$

With these definitions and preliminaries, we next state some classical results about the rotation number of non- decreasing maps, which go back to Henri Poincaré $[\mathrm{Po}]$ in the context of degree-one homeomorphisms and are not harder to prove in our slightly generalized context.

\section{Theorem A.}

- (i). For $F$ and $G$ in $\mathcal{F}^{0}(\mathbb{R})$,

$$
\begin{gathered}
F \geq G \quad \Rightarrow \quad \rho(F) \geq \rho(G), \\
F>G \& \rho(F) \text { or } \rho(G) \text { irrational } \Rightarrow \rho(F)>\rho(G) .
\end{gathered}
$$

- (ii). The rotation number, as a function $\rho: \mathcal{F}^{0}(\mathbb{R}) \rightarrow \mathbb{R}$ is continuous.

- (iii). For $F$ and $G$ in $\mathcal{F}^{0}(\mathbb{R})$, if $\rho(F)$ is irrational and $f$ has a dense orbit, then,

$$
F \geq G \quad \text { and } \quad F \neq G \quad \Rightarrow \quad \rho(F)>\rho(G) .
$$

Corollary A'. For a family $\left\{F_{\mu}\right\}$ in $\mathcal{F}^{0}(\mathbb{R})$, defined by $F_{\mu}=F_{0}+\mu$, the rotation number of $F_{\mu}$ is a non-decreasing function of $\mu$.

If $F$ is a degree-one lift, we denote by $F^{+}$its monotone upper- bound, and by $F^{-}$its monotone lower-bound (see Figure A2). In formulas:

$$
F^{+}(x)=\sup _{y \leq x}(F(y))
$$




$$
F^{-}(x)=\inf _{y \geq x}(F(y)) .
$$

Theorem B [CGT, Mi2]. For any lift $F$,

- (i). $I(F)=\left[\rho\left(F^{-}\right), \rho\left(F^{+}\right)\right]$.

- (ii). Furthermore, for each $\omega \in I(F)$, there is a non- decreasing lift $F_{\omega}$ which coincides with $F$ where it is not locally constant, and with $\rho\left(F_{\omega}\right)=\omega$.

Corollary B'. For any lift $F$, if $\omega \in I(F)$, then $F$ has a $\omega$-ordered orbit.

\section{A-3. Denjoy Theory.}

The following result about smooth maps will be used in some proofs: it is a weakened form of the "famous" result by Arnaud Denjoy [De]:

Theorem of Denjoy. For $F$ in $\mathcal{F}^{2}(\mathbb{R})$, if $\rho(F)$ is irrational then $f$ has a dense orbit.

Remark. Bohl [Boh], and independently Denjoy [De], constructed counterexamples to this statement in $\mathcal{F}^{1}(\mathbb{R})$. Such maps usually called Denjoy counterexamples.

\section{A-4. Topological entropy.}

Let $M$ be a compact metric space with distance $d, f$ an endomorphism of $M$ (i.e., a continuous map from $M$ to itself). For a positive real number $\epsilon$ and an integer $n$, the subset $S$ of $M$ is $n$ - $\epsilon$-separated if for each pair $(x, y)$ of distinct points of $S$, there is an $m$ in $\{0,1, \ldots, n-1\}$ such that

$$
d\left(f^{m}(x), f^{m}(y)\right)>\epsilon .
$$

Let $N(n, \epsilon)$ stand for the maximal cardinal (i.e., the number of elements) of an $n-\epsilon-$ separated set. We set

$$
H(\epsilon)=\limsup _{n \rightarrow \infty} \frac{1}{n} \log N(n, \epsilon) .
$$

Then the topological entropy ([AKM], [Bow]) of $f$ is

$$
h(f)=\lim _{\epsilon \rightarrow 0} H(\epsilon) .
$$


The topological entropy, originally devised as an invariant of topological conjugacy [AKM], is also a measure of the dynamical complexity of a map. By a Theorem of [MSz], for a piecewise monotone endomorphism $f$ of the circle or the interval, the topological entropy $h(f)$ is given by:

$$
h(f)=\limsup _{n \rightarrow \infty} \frac{1}{n} \log (N(n))
$$

where $N(n)$ stands for the number of maximal arcs or intervals where $f^{\circ n}$ is monotone, or the number of isolated periodic points with period $n$ of $f$. Furthermore, we have the following results:

Theorem C [BFr, Mi1]. An endomorphism of the interval has positive topological entropy if and only if it has a periodic orbit whose period is not a power of two.

Theorem D [Mi1]. An endomorphism $f$ of the interval $I$ or the circle $\mathbb{I}$ has positive topological entropy if and only if it has a horseshoe, i.e., for some $n$ and some interval $J \subset I$, or some arc $J \subset \mathbb{I}, J$ contains two disjoints subintervals $J_{0}$ and $J_{1}$ such that both $f^{n}\left(J_{0}\right)$ and $f^{n}\left(J_{1}\right)$ contain $J$. 


\section{Appendix B: Stunted families.}

Figures A3 and A4 explain respectively how to construct stunted families out of maps on the interval and the circle. Such stunted families are particularly easy to study since the topological entropy, and finer topological invariants as furnished by kneading theory [MTh], vary monotonically and continuously in each parameter ( $c f$ [DGMT]). Here we describe some examples on the interval which will help us in the study of some special circle maps.

For $S>1$ and $\mu \in\left[0,1+\frac{1}{S}\right]$, let $f_{S, \mu}$ be the endomorphism of the unit interval defined by

$$
f_{S, \mu}(x)=\left\{\begin{aligned}
& \min (S x, 1) \quad \text { if } 0 \leq x \leq \frac{\mu}{2}, \\
& \max (\min (S(\mu-x), 1), 0) \quad \text { if } \quad \frac{\mu}{2} \leq x \leq \frac{S(\mu+1)-1}{2 S}, \\
& \max (S(x-1)+1,0) \quad \text { if } \quad \frac{S(\mu+1)-1}{2 S} \leq x \leq 1,
\end{aligned}\right.
$$

This map fixes the end points, and has two extremal values (hence is bimodal), a maximum

$$
M_{S, \mu}=\min \left(1, \frac{S \mu}{2}\right)
$$

and a minimum

$$
m_{S, \mu}=\max \left(0, S \frac{\mu-1}{2}+\frac{1}{2}\right),
$$

so that the graph is like a hill followed by a valley, except in the limit cases $\mu \in\left\{0,1+\frac{1}{S}\right\}$ where the hill or valley degenerates to a point. We call plateaus the segments where the extrema are attained: in some cases, one or both plateaus may be reduced to a single point.

Fixing $S>1$ and $\mu \in[0,1]$, for $a \in\left[0, M_{S, \mu}\right]$ and $b \in\left[0,1-m_{S, \mu}\right]$ such that $a+b \geq 1$, we have a two-parameter stunted family defined by

$$
c_{S, \mu ; a, b}(x)=\left\{\begin{array}{cc}
\min \left(f_{S, \mu}(x), a\right) & \text { under the hill }, \\
\max \left(f_{S, \mu}(x), 1-b\right) & \text { in the valley }
\end{array}\right.
$$

The $(a, b)$-parameter space of $c_{S, \mu ; a, b}$ is represented in Figure A5. A nice feature of these stunted families is that, since $S>1$, one has the 
Density Property. The unions of all preimages of the plateaus (i.e., under all iterates of the map) are dense in $[0,1]$.

A proof of the density is easily supplied. A little more work is needed for the following result (see, e.g., the appendix of $[\mathrm{BMT}]$ ).

Measure Property. If any of the plateaus has non-zero length, the unions of all preimages of the plateaus (i.e., under all iterates of the map) have Lebesgue measure 1.

The combinatorial structure of an orbit is determined by the sequence of segments of monotonicity of the map visited by the orbit, as formalized in the kneading theory of Milnor and Thurston [MTh]. In order to limit the length of this paper, we shall avoid here explicit use of kneading theory (and its language), but a good knowledge of it would certainly simplify the comprehension of the rest of this section and its applications: for the kneading theory of interval maps, the reader is refereed to [MTh], and for circle maps to [AM1]. We will say the combinatorial behavior of the orbit of a plateau is plateau-avoiding if the orbit of the plateau does not hit any plateau. The following property is easy to check.

Parallel Property. Any plateau-avoiding combinatorial behavior $\beta$ of the orbit of a plateau occurs on a subset $s_{\beta}$ of the $(a, b)$ parameter space, which is a segment parallel to the $b$-axis, with one end point on the $a$-axis, for the left plateau, and a segment parallel to the $a$-axis, with one end point on the $b$-axis, for the right plateau.

We just mention here the following:

Corollary 1. For a dense subset $D$ of the $(a, b)$ parameter space, one point of a plateau belongs to a periodic orbit.

Remark. With the methods in $[\mathrm{BMT}]$, one can show that in $D$, the orbits of almost all points in $[0,1]$ converge to a periodic orbit which has (at least) one point in a plateau, and that the complement of $D$ in the $(a, b)$ parameter space, has zero Lebesgue measure. More important for our purpose is the next consequence of the Parallel Property. 
Isolated Points Property. Any pair of plateau-avoiding combinatorial behavior $\left(\beta, \beta^{\prime}\right)$ of the orbits of the plateaus occurs for at most a single point $P_{\left(\beta, \beta^{\prime}\right)}$ of the $(a, b)$ parameter space.

Combining the Parallel Property and the Isolated Points Property with the combinatorial theory for the boundary of chaos for bimodal maps ([MaT2], [MaT3], [Mu]), we get the following

Theorem $\mathbf{E}$. The region corresponding to zero topological entropy in the $(a, b)$ parameter space of $c_{S, \mu ; a, b}$, is a closed set, separated from the rest of the parameter space by a curve of length bounded from above by 2 , made of countably many pieces of straight lines crossing the gaps of a Cantor set embedded in the two- dimensional parameter space.

A construction of the curve discussed in Theorem E is shown schematically in Figure A6. Now, fixing $S$, we can form three-parameter families (with parameters $\mu, a$ and $b$ ), such that the parameter space is as represented in Figure A7- $\alpha$ when $S>3$, and as in Figure A7- $\beta$ otherwise: we denote by $\mathbf{C}_{S}$ the 3 -cell which parametrizes the family $c_{S, \mu ; a, b}$ for given $S>1$. Some two-dimensional surfaces in these cells will occur in the study of some families of circle maps. To get a result similar to Theorem E in these surfaces, we will use the following monotonicity result .

Theorem F. If some plateau-avoiding behavior of the right plateau occurs both for $c_{S, \mu ; M_{S, \mu}, b}$ and $c_{S, \mu^{\prime} ; M_{S, \mu^{\prime}}, b^{\prime}}$ for $\mu^{\prime}>\mu$, then $b^{\prime}<b$. Similarly, if some plateau-avoiding behavior of the left plateau occurs both for $c_{S, \mu ; a, m_{S, \mu}}$ and $c_{S, \mu^{\prime} ; a^{\prime}, m_{S, \mu^{\prime}}}$ for $\mu^{\prime}<\mu$, then $a^{\prime}<a$.

Proof of Theorem F. The second statement is equivalent to the first one, that we deduce from the Measure Property as follows.

- From kneading theory, we know that $c_{S, \mu ; M_{S, \mu}, b}$ and $c_{S, \mu^{\prime} ; M_{S, \mu^{\prime}}, b^{\prime}}$ have the same set of preimages of the plateaus (as labeled by the successive branches of the map where the preimages are taken). This is sufficient to conclude when $M_{S, \mu}<1$.

- When $M_{S, \mu}=1$, we conclude thanks to the following Lemma. 
Lemma $\mathbf{F}^{\prime}$. For any map of the form $c_{S, \mu ; 1, b}$, with $b<1$, and all $n \geq 0$, there is an injective map from the set of intervals which are $n^{\text {th }}$ preimages of the left plateau to the set of intervals which are $n^{\text {th }}$ preimages of the right plateau, and the injection is strict for some values of $n$.

(Q.E.D. Theorem F.)

Proof of Lemma F'. From the already mentioned property in kneading theory, we choose to work with the map $c_{3, \frac{2}{3} ; 1,1}$ for convenience of the exposition, without loss of generality. Under this map, the two turning points, $\frac{1}{3}$ and $\frac{2}{3}$, have symmetric sets of preimages, and we only need to show that for any map $c_{3, \frac{2}{3} ; 1, b}$ with $b<1$, the left turning point has lost at least as many preimages as the right turning point, at each generation, and sometimes more.

We will only consider preimages of $\frac{1}{3}$ and $\frac{2}{3}$ to the left of $\frac{2}{3}$, preimages on the other side of $\frac{2}{3}$ being treated similarly. For each $n>0$, let $x_{n, N(n)}<x_{n, N(n)-1}<\ldots<x_{n, 1}$, with $x_{n, 1}<\frac{2}{3}$, be the preimages of $\frac{2}{3}$, up to the $n^{\text {th }}$ generation, and such that $c_{3, \frac{2}{3} ; 1,1}\left(x_{n, i}\right)<$ $1-b$, and let $m_{n, i} \leq n$ be the generation index of $x_{n, i}$, i.e., $c_{3, \frac{2}{3} ; 1,1}^{m_{n, i}}\left(x_{n, i}\right)=\frac{2}{3}$. Let $I_{n, 1}$ stand for the interval between $x_{n, 1}$ and $\frac{2}{3}$. Then $c_{3, \frac{2}{3} ; 1,1}^{m_{n, 1}}\left(I_{n, 1}\right)$ contains the other turning point $\frac{1}{3}$, so that $I_{n, 1}$ contains a preimage of $\frac{1}{3}$ with generation index at most $m_{n, 1} \leq n$. Similarly, for each $i \in\{2,3, \ldots, N(n)\}$, the interval $I_{n, i}=\left(x_{n, i}, x_{n, i-1}\right)$ contains a preimage of $\frac{1}{3}$ with generation index at $\operatorname{most} \max \left(m_{n, i}, m_{n, i-1}\right) \leq n$, as we show now to conclude the proof of injectivity.

- either $m_{n, i}=m_{n, i-1}$, in which case the paths of the orbits of $x_{n, i}$ and $x_{n, i-1}$ until $\frac{2}{3}$ is reached have to be different: the only way this can occur is the image of $I_{n, i}$, under $c_{3, \frac{2}{3} ; 1,1}^{q}$ for some $q<m_{n, i}$, contains $\frac{1}{3}$ since $x_{n, i}$ and $x_{n, i-1}$ are consecutive.

- or $m_{n, i} \neq m_{n, i-1}$, that we rewrite as $m_{n, j}<m_{n, k}$ : then, when the orbit of $x_{n, k}$ reaches $\frac{2}{3}$, the orbit of $x_{n, j}$ has reached the fixed point 0 , and again, some image of $I_{n, i}$ contains $\frac{1}{3}$.

To show that the injection is strict for some values of $n$, it remains to show that for some values of $n$, there is a preimage of $\frac{1}{3}$ with generation at most $n$ to the left of $x_{n, N(n)}$. Notice then that either 
- (i) $x_{n+1, N(n+1)-1}=x_{n, N(n)}$, or

- (ii) $m_{n+1, N(n+1)-1}=n+1$.

In case (ii), we conclude as before that the image of $I_{n+1, N(n+1)}$, under $c_{3, \frac{2}{3} ; 1,1}^{q}$ for some $q<m_{n+1, N(n+1)}$, hence $q \leq n$, contains $\frac{1}{3}$.

In case (i), if $x_{n+1, N(n+1)}$ is not the preimage of $x_{n, N(n)}$, we conclude as in case (ii), and $x_{n+1, N(n+1)}$ cannot be the preimage of $x_{n, N(n)}$ for all $n$ 's, since such monotonic sequences would have to accumulate on one of the end points of the interval, and the leftmost preimage of order $n$ of $\frac{1}{3}$ is to the left of the leftmost preimage of $\frac{2}{3}$ or order $n$ or less.

(Q.E.D. Lemma F'.)

\section{Remarks.}

- Theorem F has just been stated here with the generality required for the study of the boundary of chaos: to suppress the words "plateau- avoiding" in this statement, one can use the methods in $[\mathrm{BMT}]$. The plateau-avoiding case is simpler since all preimages of both plateaus are pairwise disjoint.

- If some plateau-avoiding behavior $\beta$ of the right plateau occurs for $c_{S, \mu_{\beta}(b) ; 1, b}$ (respectively if some plateau- avoiding behavior $\beta$ of the left plateau occurs for $\left.c_{S, \mu_{\beta}(a) ; a, 1}\right)$ it follows from the proofs of Lemma $\mathrm{F}^{\prime}$ and Theorem $\mathrm{F}$ that $\mu_{\beta}(b)$ (respectively $\mu_{\beta}(a)$ ) is a linear function. Combining this with the previous remark, the same linear property holds true if $\beta$ corresponds to a periodic behavior of an end-point of a plateau. 


\section{Appendix C: Computation of Boundaries of Some Regions in Parameter Space.}

Since the maps discussed in this paper are piecewise linear, it is possible to find exact equations for the boundaries of the various parameter plane regions discussed in the previous parts of the paper, although in most cases obtaining such equations in a simplified form is not trivial. In this appendix, we will describe the exact equations of most of the boundaries, especially for the tip family, and give a brief indication of how the equations were obtained. The derivations of the boundary equations are elementary and come from a few simple facts about circle maps $f$ and their lifts $F$, such as the following where we have identified the circle with the interval $[0,1), x \in[0,1)$, and $F$ is the particular lift of $f$ satisfying $F(0) \in[0,1)$ :

-i) $x$ belongs to a $p / q$-cycle of the circle map $f$ if and only if $F^{q}(x)=x+p$.

-ii) On the left (respectively, right) boundary of $A_{p / q}$ the graph of the $q^{t h}$ iterate $F^{q}$ of the appropriately chosen lift is tangent to the line $y=x+p$ from below (above), in the $(\Omega, U)$ parameter plane.

-iii) On the left (respectively, right) boundary of $A_{p / q}$, a $p / q$-cycle of a tip or plateau map must contain the first (respectively, last) turning point of the map. These turning points are the first and last points in Figure 1 where the slope is undefined.

-iv) If $x$ is a member of a stable (attractive) $p / q$-cycle of $f$ then at $x$ the slope of $F^{q}$ is less than 1 in magnitude.

\section{C-1. Boundaries of $A_{p / q}$ for the Tip Maps.}

In the sections below we will obtain the boundary equations of each connected com-

ponent of the interior of the sausage-like structure $A_{p / q}$. The points that separate these connected components are called nodes. The equations representing the boundaries of $A_{p / q}$ will change from one component to another of $\operatorname{int}\left(A_{p / q}\right)$. For each $p / q$ the components of $\operatorname{int}\left(A_{p / q}\right)$ belong to disjoint horizontal strips in the $(\Omega, S)$ plane. Since the number of components is finite, exactly one of them is unbounded. This unbounded component, which 
is the component that intersects the critical line, we call the top component of $\operatorname{int}\left(A_{p / q}\right)$. The top node is the node that separates the top component from the rest of $A_{p / q}$.

\section{C-1.1. Nodes.}

In this section we will find the heights of the nodes in the $(\Omega, S)$ parameter plane. As discussed in the proof of Theorem 4 in $\S 7$, the tongue $A_{p / q}$ has zero width in the $(\Omega, S)$ parameter plane, for fixed $\delta$, when the corresponding circle map $f$ satisfies $f^{q}=\operatorname{Id}$ (the identity map on the circle). Such a point $(\Omega, S)$ in the parameter plane is a node, except at the foot of the tongue where $S=1$. Thus the nodes are the points that separate the different "links" of the sausage-like structure $A_{p / q}$.

By setting the slope of $f^{q}$ equal to 1 , as in the proof of Theorem 4, one comes up with the polynomial equation that must be satisfied by the parameter $S$ at a node:

$$
S^{q-k}[1-S(1-\delta)]^{k}-\delta^{k}=0
$$

for $k=1, \ldots,\lceil\delta q\rceil-1$, where $\lceil x\rceil=\operatorname{ceiling}(x)=$ the least integer greater than or equal to $x$.

At a node of $A_{p / q}$ the $p / q$-cycle for $t_{\Omega, S ; \delta}$ must contain both turning points of the map. As an illustration of how the nodes occur, consider Figure C1, which shows how the members of a typical $2 / 5$-cycle vary as $(\Omega, S)$ descends along the left boundary of $A_{p / q}$ from the critical line $S=S_{c}$ to the foot of the tongue at $S=1$. In this figure, $\delta \approx 1 / 2$, the circle is represented as the interval $[-1 / 2,1 / 2)$, and the portion of the circle between the turning points $t_{1}=-t_{0}=(1-\delta) / 2$ is the big slope region of the map. When a member of the $2 / 5$-cycle coincides with $t_{0}$ a node occurs. This figure illustrates the proof of Theorem 4 and also illustrates the reason that the number of nodes in $A_{p / q}$ is $\lceil\delta q\rceil-1$.

Equation $(\mathrm{C} 1)$ can be used to determine the nodes of $A_{p / q}$ to any desired degree of accuracy with the help of Newton's Method, and for a given $S$ by testing the left side of (C1) for $k=0,1, \ldots$, one can determine which link of the sausage is cut by the horizontal line at $S$. Figure C2 illustrates typical locations of the turning points $t_{0}, t_{1}$ as well as the big slope region of a tip map. 


\section{C-1.2. Further properties of the nodes.}

A summary of other properties of the set of nodes in $A_{p / q}$ is given below. These properties can be proved by elementary methods and are illustrated by the various figures in this section (see especially Figures C3 to C15, which will be discussed in detail later).

Nodes in relation to the critical line:

All nodes of $A_{p / q}$ are below the critical line.

Nodes for even q:

If $\delta>1 / 2$ and $S_{0}=\delta /(1-\delta)$ then all $A_{p / q}$ with even $q$ have nodes on the line $S=S_{0}$. In fact, the $p / q$ sausage has its $\frac{q}{2}$ th node on this line, counting down from the top node. If $\delta \leq 1 / 2$ there is no such $S_{0}$.

Rows of nodes:

Assume $q>1$ and let $t_{1}=-t_{0}=(1-\delta) / 2$. Below the critical line in the $(\Omega, S)$ plane, the line $\Omega+S t_{1}=t_{0}+1$ intersects $A_{p / q}$ at nodes only. Similarly, there are rational curves $\Omega=P(S) / Q(S)$ of all degrees $(k$ over $k-1)$ that intersect the sausages below the critical line only at nodes.

These rational curves are obtained by solving $T_{\Omega, S ; \delta}^{q}\left(t_{1}\right)=t_{0}+p$ for $\Omega$ where $q=$ $1,2, \ldots$ and where $T$ is the lift of $t$. Two such rational curves through nodes are drawn in Figure C16 for $q=1,2$ and they can also be seen in some of our other figures showing groups of $p / q$-tongues.

How the nodes vary with $\delta$ :

Suppose $p / q \in[0,1)$ is in lowest terms. Then:

a) For small $\delta, R_{p / q}$ has no nodes.

b) For $\delta$ sufficiently close to $1, R_{p / q}$ has $\lceil q\rceil-1$ nodes, i.e., it has its full allowance of nodes and will get no more.

c) As $\delta \rightarrow 1$, all nodes of $R_{p / q}$ approach the critical line. (Recall that the height $S_{c}$ becomes unbounded as $\delta \rightarrow 1$. The upper nodes (those with node number $k<q / 2$ ) approach the critical line absolutely: $S_{\text {node }}-S_{c} \rightarrow 0$ as $\delta \rightarrow 1$ while the other nodes approach the critical line relatively: $\left(S_{\text {node }}-S_{c}\right) / S_{c} \rightarrow 0$ as $\delta \rightarrow 1$. As $\delta \rightarrow 1$, the $\Omega$-coordinates of all nodes approach 1/2, as illustrated in Figures C3 to C15. 


\section{C-1.3. Boundaries of $A_{p / q}$ above the top node.}

We now proceed to find equations of the exact boundaries of $A_{p / q}$ for the tip family. For simplicity we first discuss these boundaries above the top node. The top component of $\operatorname{int}\left(A_{p / q}\right)$ intersects the critical line, so that the boundary equations obtained in this section will also yield the exact endpoints of the stability intervals on the critical line.

Below the critical line the equations of the left and right boundaries of $A_{p / q}$ will change from link to link of the sausage structure. In order more easily to understand how the boundary equations are obtained we consider in Figure C2 a typical lift of the tip family. The particular lift shown corresponds to a parameter pair $(\Omega, S)$ above the critical line, where the small slope $s=[1-S(1-\delta)] / \delta$ is negative. Above the first node, the tip family boundary equations for $A_{p / q}$ will be the same whether $(\Omega, S)$ is below or above the critical line.

The three linear functions $f_{1}, f_{2}, f_{3}$ that we will use to define a lift of the tip map $t_{\Omega, S ; \delta}$, as shown in Figure $\mathrm{C} 2$, are:

$$
\begin{aligned}
& f_{1}(x)=\Omega+s(x+1 / 2)-1 / 2, \\
& f_{2}(x)=\Omega+S x \\
& f_{3}(x)=\Omega+s(x-1 / 2)+1 / 2,
\end{aligned}
$$

where for convenience in the following discussion we will now identify the circle with the interval $[-1 / 2,1 / 2)$. (We could use any interval of length 1 ; this one seems most convenient.) To help understand the three functions $f_{1}, f_{2}, f_{3}$, recall that the map $t_{\Omega, S ; \delta}$, for fixed $\delta$, consists of two linear parts, one with the "big slope" $S$ and the other with the "small slope" $s$. The function $f_{2}$ represents the "big slope" part and the other two functions $f_{1}$ and $f_{3}$ represent the "small slope" part of $t_{\Omega, S ; \delta}$. No matter where $(\Omega, S)$ lies in the sausage structure $A_{p / q}$, we will be able to represent $t_{\Omega, S ; \delta}$ and all its iterates using the three functions $f_{1}, f_{2}, f_{3}$ along with the decrement function described below. Note that $f_{3}$ is just a shifted version of $f_{1}$ since $f_{3}(x) \equiv f_{1}(x-1)+1$.

The above three functions will now be used to find the boundary equations of $A_{p / q}$. We first consider the left boundary of $A_{p / q}$ above the top node. On the left boundary, the 
following equation must be satisfied:

$$
t_{\Omega, S ; \delta}^{q}\left(t_{1}\right)=t_{1}
$$

where appropriate choices among $f_{1}, f_{2}, f_{3}$ must be made for each of the iterates in $t^{q}$. The thing that distinguishes one link of the sausage from the other links (see Figure C1) is the number of $p / q$-cycle points that are outside the big slope region of the circle map. To be more precise, if the circle is represented by the interval $[-1 / 2,1 / 2)$ then the "big slope" region is the interval $\left(t_{0}, t_{1}\right]$ in Figure $\mathrm{C} 2$. When no points of the $p / q$-cycle are outside this interval then the parameter pair $(\Omega, S)$ is in the top link, which includes the part of $A_{p / q}$ above the critical line. When k points of the cycle are outside the interval then $(\Omega, S)$ is in the $k^{t h}$ link from the top, where $k=1, \ldots,\lceil q \delta\rceil-1$ as shown by Theorem 4 .

In order to simplify the boundary equation, we introduce the decrement function:

$$
d(x)=x-1 .
$$

Since the values of the lift wrap around the circle $p$ times during a $p / q$-cycle, the left side of equation (C3) is represented by $q$ copies of $f_{2}$ interspersed with $p$ copies of the decrement function $d$ to indicate wrapping around the circle $p$ times. Hence the equation of left boundary of the top link of $A_{p / q}$ for $q>1$ is given schematically by

$$
f_{2} \cdots f_{2} d f_{2} \cdots f_{2} d f_{2}\left(t_{1}\right)=t_{1}
$$

where the left side of (C4) is a composite of $q$ copies of $f_{2}$ and $p$ copies of $d$. The positions of the $d$ 's are given by the following rule: Counting from right to left in $(\mathrm{C} 4)$, we insert a " $d$ " to the left of the $i$ th $f_{2}$ for each $i$ such that

$$
0<i p \quad(\bmod q) \leq p \quad(i=1, \ldots, q-1)
$$

For the case $q=1$ the left boundary equation is simply $f_{2}\left(t_{1}\right)=t_{1}$, which is easily solved for $\Omega$ to obtain

$$
\Omega=-(S-1) t_{1}=\Omega_{\text {left }}(S, 0 / 1, \delta) .
$$


Equation (C4) contains the quantities $\Omega, S, \delta$ [recall $t_{1}=(1-\delta) / 2$ ], $p$, and $q$. By using properties of the simple functions $f_{2}$ and $d$ it is relatively straightforward to solve the equation (C4) for $\Omega$, with the following result:

Left tip boundary above the top node:

For $q>1$ and $p / q$ in lowest terms,

$$
\Omega=\Omega_{\mathrm{left}}(S, p / q, \delta)=(S-1)\left[\frac{N_{\mathrm{left}}(S, p / q)}{S^{q}-1}-t_{1}\right]
$$

where

$$
N_{\text {left }}(S, p / q)=\sum_{\substack{i=1 \\ 0<i p}}^{q-1} S^{q-i}
$$

The right boundary of $A_{p / q}$ can be obtained in a similar manner to the above. On the right boundary, a $p / q$-cycle of the tip map contains the other turning point, $t_{0}=-(1-\delta) / 2$, which leads to:

$$
d f_{2} \cdots f_{2} d f_{2} \cdots f_{2} d f_{2} \cdots f_{2}\left(t_{0}\right)=t_{0}
$$

where the left side of (C6) is a composite of $q$ copies of $f_{2}$ and $p$ copies of $d$. , and where, counting from right to left in (C6), we insert a " $d$ " to the left of the $i$ th $f_{2}$ for each $i$ such that

$$
0 \leq i p \quad(\bmod q)<p \quad(i=2, \ldots, q)
$$

Solving equation (C6) for $\Omega$, again after some straightforward but nontrivial computations, gives the equation for the right boundary of $A_{p / q}$ for the tip family above the top node:

Right tip boundary above the top node:

For $q>1$ and $p / q$ in lowest terms,

$$
\Omega=\Omega_{\text {right }}(S, p / q, \delta)=(S-1)\left[\frac{N_{\text {right }}(S, p / q)}{S^{q}-1}-t_{0}\right],
$$

where,

$$
N_{\text {right }}(S, p / q)=\sum_{\substack{i=2 \\ 0 \leq i p}}^{q} S^{q-i}=N_{\text {left } q)<p}(S, p / q)+1-S^{q-1} .
$$


The boundary equations (C5) and (C7) are valid for the tip family version of $A_{p / q}$ anywhere above the top node, including on and above the critical line. By subtracting equation (C5) from (C7), for example, one obtains the exact equation for the length of the $p / q$-stability intervals on the critical line, which was given in Section 4 of this paper and used in the proof of Theorem 5:

Length of $p / q$-stability interval on the critical line:

$$
\left|L_{p / q}^{\circ}\right|=\Omega_{\text {right }}-\Omega_{\text {left }}=\frac{(S-1)^{2}}{S\left(S^{q}-1\right)},
$$

where on the critical line, $S=S_{c}=1 /(1-\delta)$. Equation (C8) for the width of $A_{p / q}$ for the tip family, at height $S$ in the parameter plane, is valid for any $S$ above the top node of $A_{p / q}$, including on and above the critical line.

When $q=1, A_{p / q}$ has no nodes, since then $\lceil q \delta\rceil-1=0$, and hence the complete left or right boundary of a $0 / 1$ or $1 / 1$ tip family tongue is easily found in a manner similar to the way we found the left boundary $\Omega=\Omega_{\text {left }}(S, 0 / 1, \delta)$. The resulting $0 / 1$ and $1 / 1$ boundary equations are:

Complete tip family boundaries for $q=1$ :

$$
\begin{aligned}
& \Omega=\Omega_{\text {left }}(S, 0 / 1, \delta)=-(S-1) t_{1}, \\
& \Omega=\Omega_{\text {right }}(S, 0 / 1, \delta)=-(S-1) t_{0}, \\
& \Omega=\Omega_{\text {left }}(S, 1 / 1, \delta)=-(S-1) t_{1}+1, \\
& \Omega=\Omega_{\text {right }}(S, 1 / 1, \delta)=-(S-1) t_{0}+1,
\end{aligned}
$$

where $t_{1}=(1-\delta) / 2$ and $t_{0}=-t_{1}$.

Note that each of these lines in the $(\Omega, S)$ plane has a slope of $\pm 2 /(1-\delta)$ when $S$ is expressed as a function of $\Omega$.

\section{C-1.4. Boundaries of $A_{p / q}$ below the top node.}

In this subsection we briefly describe the exact boundary equations for $A_{p / q}$ below the top node.

For a given $S>1$ it is easy to determine which link of the $p / q$-sausage is cut by the horizontal line at $S$ : just test the left side of equation (C1) for $k=1,2, \ldots,\lceil q \delta\rceil-1$; the 
first $k$ that gives a value $\leq 0$ tells us that the height $S$ corresponds to the $k$ th link from the top, where the part above the top node is link 0 .

For every one of the sausage links one can write the left and right boundary equations

$$
t_{\Omega, S ; \delta}^{q}\left(t_{i}\right)=t_{i}, \quad(i=1,0 \text { respectively },)
$$

in forms similar to $(\mathrm{C} 4)$ and $(\mathrm{C} 6)$. The fact that on the boundary of link $k, k$ of the $p / q$ cycle members have escaped from the big slope region of the circle map $t_{\Omega, S ; \delta}$, means that $k$ of the " $f_{2}$ " s in (C4) and (C6) must be replaced by " $f_{1}$ " s or " $f_{3}$ "s. It is straightforward to replace (C4) and (C6) with versions that work for the boundaries of the $k$ th link from the top for arbitrary $k$ in the admissible range $0,1, \ldots,\lceil q \delta\rceil-1$. We will not dwell on the equations for arbitrary $k$, but will simply illustrate with the following example.

\section{Example:}

The right boundary equations for the 3 links of $A_{2 / 5}$, are:

$$
\begin{array}{ll}
k=0: & d f_{2} f_{2} d f_{2} f_{2} f_{2}\left(t_{0}\right)=t_{0} \\
k=1: & d f_{2} f_{2} d f_{3} f_{2} f_{2}\left(t_{0}\right)=t_{0} \\
k=2: & d f_{3} f_{2} d f_{3} f_{2} f_{2}\left(t_{0}\right)=t_{0} .
\end{array}
$$

By writing the specific formulas of each of the " $f$ " and " $d$ " functions, one can solve each of these equations explicitly for $\Omega$ in terms of $S$ and $\delta$. For example, the middle equation above, when solved for $\Omega$, gives the right boundary of link 1 :

$$
\Omega=\Omega_{\text {right }}=\frac{\delta(1+\delta)+(1+\delta) S^{2}-(1-\delta) S^{3}+(1-\delta) S^{4}-(1-\delta)^{2} S^{5}}{2\left[\delta+\delta S+(1+\delta) S^{2}+\delta S^{3}-(1-\delta) S^{4}\right]}
$$

For the purpose of generating computer-drawn curves of the left and right boundaries of the tip family tongues $A_{p / q}$, we have written a general computer algorithm that for a given $S \geq 1$, solves the left and right boundary equations corresponding to (C4) and (C6) for the appropriate link. The algorithm finds the exact (up to computer accuracy) values of $\Omega$ on the left and right boundaries for a given $S$ by recursively computing the left side of the equation as a affine function of $\Omega$ and then solving the affine equation for 
$\Omega$. This computer algorithm has been used to generate accurate drawings of the left and right boundaries of many of the tongues in this paper, including parts of the boundaries of the stability regions shown later in Appendix C.

\section{C-1.5. The unbounded part of $A_{p / q}$.}

As explained in Section C-1.3, equations (C5) and (C7) give the left and right boundaries, respectively, of the tip family $A_{p / q}$ above the top node, which also includes the portion on and above the critical line. So we have nothing more to say about the left and right boundaries above the line, except to note that in equations (C5) and (C7) the two numerators $N_{\text {left }}$ and $N_{\text {right }}$ each are polynomials in $S$ of degree less than $q$. Hence equations (C5) and (C7) imply that for large $S$, the right and left boundaries of $A_{p / q}^{+}$in the $(\Omega, S)$ plane are asymptotic to straight lines with slope $\pm 2 /(1-\delta)$. Thus, all tongue boundaries are asymptotically parallel as $S \rightarrow \infty$.

\section{C-1.6. Boundaries of other $p / q$ regions above the critical line.}

By considering the slopes of a tip map $t_{\Omega, S ; \delta}$ and its $q$ th iterate $t^{q}$, it is fairly straightforward to deduce other statements concerning the exact borders of the various regions previously discussed in this paper. These properties are summarized below, where for fixed $\delta, A_{p / q}^{\text {stab }}=$ the $p / q$-stability region, $A_{p / q}^{s t a b^{\prime}}=$ the extended stability region where $t_{\Omega, S ; \delta}$ has either a stable $p / q$-cycle or a $p / q$-ordered chaotic attractor (as defined just before Theorem 18 ), and $L_{p / q}=$ the region where $t_{\Omega, S ; \delta}$ has the unique rotation number $p / q$. Some of these regions were shown in Figures 7, 13, 14, 15 and 18. The boundaries in those figures were obtained from the equations in this appendix.

The heights of the regions:

Let $S=S_{\max }(q), S_{\max }^{\prime}(q)$, and $S_{\text {tip }}(q)$ be the heights in the $(\Omega, S)$ plane of the regions $A_{p / q}^{s t a b}, A_{p / q}^{s t a b^{\prime}}$, and $L_{p / q}$, respectively. Then:

a) The three heights satisfy, respectively, the following three polynomial equations:

$$
\begin{array}{ll}
S_{\max }: & (1-\delta) S^{q}-S^{q-1}-\delta=0, \\
S_{\max }^{\prime}: & (1-\delta) S^{q+1}-S^{q}-S+1=0,
\end{array}
$$


$S_{\mathrm{tip}}: \quad(1-\delta) S^{q+1}-S^{q}-(2-\delta) S+2=0$.

b) $S_{\text {tip }}>S_{\max }^{\prime}>S_{\max }>S_{c}$.

c) All three heights decrease with $q$ and as $q \rightarrow \infty$ they approach the critical line $S=S_{c}=1 /(1-\delta)$ exponentially fast in q.

Of course, with the help of Newton's method or other numerical procedures, the above three heights can easily be computed very accurately and quickly.

The top boundary of $L_{p / q}$ :

The left (respectively, right) boundary of $L_{p / q}$ above the critical line, is the limit of the right (left) boundaries of the neighboring $A_{\omega}$ as $\omega \rightarrow p / q$ from the left (right). Since in practice these neighboring boundaries converge rather quickly with $\omega$, one can use the results of Section C-1.3 to draw quite accurate boundaries of $L_{p / q}$ above the critical line. The left and right boundaries of $L_{p / q}^{+}$intersect at height $S=S_{\text {tip }}(q)$, so that the top of $L_{p / q}$ is somewhat "ice cream cone" shaped, as is seen in Figure 7. Of course, below the line, $L_{p / q}$ is the same as $A_{p / q}$.

Top boundaries of $A_{p / q}^{s t a b}$ and $A_{p / q}^{s t a b^{\prime}}$ :

$A_{p / q}^{s t a b}$ is the portion of $A_{p / q}$ bounded above by the horizontal line $S=S_{\max }$ described above. Similarly, $A_{p / q}^{s t a b^{\prime}}$ is the portion of $A_{p / q}$ bounded above by the horizontal line $S=S_{\max }^{\prime}$.

Boundary of the p/q-topologically regular region:

From Section 9 of this paper, the part of $A_{p / q}$ that has zero topological entropy is $A_{p / q}^{s t a b} \cap$ $L_{p / q}$, so this region can be accurately plotted by using boundaries determined in the above discussions.

With the above properties we can draw the various tip family $p / q$-regions very accurately along with their unions and intersections. These properties were used to generate Figures C3 to C15 showing the exact boundaries of $A_{p / q}^{s t a b}$ as $\delta$ increases from 0.1 to 0.9999. These figures illustrate many of the concepts discussed in this paper.

\section{C-1.7. The zipper theorems.}


We call Theorems 17 and 18 the Zipper Theorems because of the way they indicate that the Farey neighbors of $A_{p / q}^{s t a b}$ and $A_{p / q}^{s t a b^{\prime}}$ unzip from each of them as $\delta$ decreases. These theorems are represented accurately in Figures 15abc and 18 of the main body of this paper, using the boundary equations and algorithms described above.

\section{C-1.8. Monte Carlo plotting of $A_{p / q}^{s t a b}$.}

One can also obtain reasonably accurate plots of the bifurcation structure of our families by using a numerical Monte Carlo algorithm such as the following, where color $C(p, q)$ is a predefined color table [normally $C(p, q)=q]$ :

- For each $(\Omega, U)$ in a rectangle, do:

- Choose $x$ randomly

. $\quad$ Repeat $x=f(x) M$ times

. For $q=1$ to $q_{\max }$

$$
\text { . If }\left|f^{q}(x)-x\right|<\epsilon \text { then }
$$

- $\quad$ Find the winding number $p / q$

. $\quad$ Plot the point $(\Omega, U)$ in $\operatorname{color} C(p, q)$

. $\quad$ Exit the q loop

. $\quad$ End if

- End the q loop

- End the do loop

This algorithm was used to plot Figure 10 for the plateau family regions $A_{p / q}^{\text {stab }}, q \leq 5$, $\delta=1 / 2$. It was also used for Figure C17 ( $A_{p / q}^{s t a b}$ for the tip family, $\left.q \leq 7, \delta=1 / 2\right)$ which should be compared with the exact version in Figure C7. The Monte Carlo algorithm gives good approximations of all the exact versions in Figures C3-C15.

\section{C-2. Plateau Family Boundaries above the Critical Line.}

In this section we will describe the boundaries of the various regions above the critical line for the plateau family.

Some of the $p / q$ regions for the plateau family are considerably more complicated than for the tip family, because of the flat spots (plateaus) on the graph of the lift $P_{\Omega, H ; \delta}$ and 
its iterates. These flat spots allow infinite sequences of period doubling, tripling, etc., as is the case with the standard sine family and the logistics family.

We do not have exact descriptions of the upper boundaries for the plateau stability and topologically regular $p / q$-regions, as we did for the tip family. However some of the other regions are actually simpler to describe exactly than for the tip family and can be obtained by elementary methods. To understand fully the structure of all parts of the stability regions, one needs to consult works on symbolic dynamics, such as [MaT1-3].

Following is a summary of our results about the plateau family above the critical line. The formulas below come in an elementary manner from the slopes in the plateau maps $\left( \pm S_{c}\right.$ and 0$)$ and from our exact equations in Section $\mathrm{C}-1$ about the boundaries of the $p / q$-intervals on the critical line.

Assume $\delta$ is fixed, $0<\delta<1$ and that $p / q$ is in lowest terms.

Top Boundary of $A_{p / q}$ :

The top of $A_{p / q}$ for the plateau family in the $(\Omega, H)$ plane is the horizontal line

$$
H=H_{\max }=\frac{\delta}{4(1-\delta)} .
$$

Side boundaries of $A_{p / q}$ :

The left and right boundaries of $A_{p / q}$ are the straight lines:

$$
\begin{gathered}
H=H_{\text {left }}(\Omega)=\left(\Omega_{L}-\Omega\right) / \delta, \\
H=H_{\text {right }}(\Omega)=\left(\Omega-\Omega_{R}\right) / \delta,
\end{gathered}
$$

where $\left(\Omega_{L}, \Omega_{R}\right)$ is the $p / q$-stability interval on the critical line for the tip family. $\Omega_{L}$ and $\Omega_{R}$ are given exactly by (C5) and (C7) in Section C-1 with $S=S_{c}=1 /(1-\delta)$.

The next result follows easily by taking a limit of boundaries of the neighboring regions. All of these boundaries have slope $\pm 1 / \delta$, from the previous property.

The $L_{p / q}$ cone:

For the plateau family, $L_{p / q}^{+}$is shaped like an inverted cone (or a tent) with straight line sides having the equations:

$$
H=\left(\Omega-\Omega_{L}\right) / \delta \quad(\text { left })
$$




$$
H=\left(\Omega_{R}-\Omega\right) / \delta \quad(\text { right })
$$

When $\delta<1-3^{-1 / q}$, the cone is truncated at height $H=H_{\max }$.

The stability region $A_{p / q}^{+, s t a b}$ :

For the plateau family, the stability region $A_{p / q}^{+, s t a b}$ is composed of several disjoint parts, as follows. These regions are shown in the numerically generated Figure 10 and schematically in Figure C18.

a) a lower, winged-shaped part with bottom boundary $H=0$, left and right boundaries the same as $A_{p / q}$ above, and top boundary composed of the two lines

$$
H= \pm\left(\Omega-\Omega_{\text {mid }}\right) \frac{H_{\max }}{\left(\Omega_{R}-\Omega_{L}\right) / 2+\delta H_{\max }}
$$

where $\Omega_{\text {mid }}=\left(\Omega_{L}+\Omega_{R}\right) / 2$,

b) an infinite number of swallow-like period multiplying regions with rotation numbers $(k p) /(k q)$ for $k=2,3, \ldots$, which lie above the "wing" described in a) above.

c) a finite number of swallow-like $p / q$-regions that lie outside $L_{p / q}^{+}$and outside the period multiplying region. See Figure 10 for a 2/5-swallow of this type.

\section{Location of swallow tips:}

All of the above wing and swallow-like $(k p) /(k q)$-regions have the tips of their wings and tails on the line $H=H_{\max }$.

As we noted earlier, understanding the structure of these swallows requires knowledge of the topology of symbol sequence dynamics but is fairly well understood. In [MaT1-3] it is explained how the boundary of topological chaos is squeezed between the regions of period $2^{k} q(k=1,2, \ldots)$ and those of period $m 2^{k} q$ where $m$ is odd. As a result the $p / q$ region of topological regularity includes the cone $L_{p / q}^{+}$chopped off on the top in a (fractal) jagged way somewhat as suggested by Figure C19.

Note that the regions for the plateau family, above the critical line, preserve most of the topological features of the regions for the classical sine circle family. Compare, for example, the topological structures of $p / q$-regions for the plateau family in Figure 10 with 
those for the sine family in Figure C20. (Both figures were generated numerically.) One can see that for each structure that appears in Figure 10 a corresponding, similar but distorted, version appears in Figure C20, although those in Figure C20 may be difficult to see because the sine $p / q$-wings and swallows appear to shrink faster with $q$ than do the plateau versions. Also note that the plateau wings and swallows are bounded, while the corresponding sine versions have infinitely long wings and tails. 


\section{Appendix D: Measures below the Critical Line.}

A natural question to ask is, for $1<S_{0}<S_{c}=1 /(1-\delta)$, what is the one-dimensional Lebesgue measure of the intersection of the horizontal line $S=S_{0}$ with the union of the $A_{p / q}, 0 \leq p / q \leq 1$ ? Since the "sausages" do not intersect each other below the critical line, the sum of these interval lengths on the line $S=S_{0}$, for $0 \leq \Omega \leq 1$, is no larger than 1. But is it less than 1 or is it equal to 1? As was remarked at the end of Section 7, for "nice" smooth families of circle maps, including the standard sine family, this measure is known [He1] to be less than 1. But for certain families of maps that are continuous on the circle and $C^{2}$ smooth except at one point, the measure is known to be equal to 1 [VK], even below the critical line: i.e., the set of $\Omega$ corresponding to irrational winding numbers has zero measure for any non-zero value of the nonlinearity parameter $S-1$. Our maps resemble those of [VK] but have two points of nonsmoothness (rather than one point), namely, the turning points, $(1 \pm \delta) / 2$. Based on the numerical results we describe below, we believe that for our family below the critical line the measure of the rational ("mode-locked") intervals is less than 1.

Using the algorithm described in Section C-1 of the Appendix, we have written a computer code that, for given values of $\delta, S=S_{0}<S_{c}$ and $q_{\max }$, finds the "exact" (up to computer accuracy) endpoints of the intersection of the line $S=S_{0}$ with all $A_{p / q}$ for $q=$ $1, \ldots, q_{\max }$ and $p=0,1, \ldots, q$ where $p$ and $q$ are relatively prime. The program then sums the lengths of these intervals on the line $S=S_{0}$. The resulting approximate measures for various values of $S_{0}, 1 \leq S_{0} \leq S_{c}=1 /(1-\delta)$, are shown in Figure D1. The measures in

Figure D1 are a result of summing as many as 12,232 interval lengths $\left(q_{\max }=200\right)$ for each value of $\mathrm{S}$, using 29 significant decimal digits of precision.

It should be emphasized that the results in Figure D1 are preliminary numerical results and are not conclusive evidence that the measure of the mode locked intervals below the critical line are less than 1 . More rigorous work is needed on this question.

\section{REFERENCES.}

[AKM] R.L. Adler, A.G. Konheim, and M.H. Mc Andrew, "Topological entropy", Trans. 
Amer. Math. Soc. 114 (1965) 309-319.

[AM1] L. Alsedà and F. Manõsas, "Kneading theory and rotation intervals for a class of circle maps of degree one", Nonlinearity 3 (1990) 413-452.

[AM2] L. Alsedà and F. Manõsas, "The monotonicity of the entropy for a family of degree one circle maps", Preprint Barcelona (1992).

[Ar] V.I. Arnold, "Small denominators I, Mappings of the circle onto itself", Izv. Akad. Nauk SSSR Ser. Mat.25 (1961) 21-86, (English translation Transl. Amer. Math. Soc. 46 (1965) 213-284).

[Ar2] V. I. Arnold, "Cardiac arrhythmias and circle mappings," Chaos 1 (1991) 20-25; this article is a previously unpublished section of Arnold's 1959 diploma dissertation which was omitted in the published reference $[\mathrm{Ar}]$.

[Bak] P. Bak, "The Devil's Staircase," Physics Today, (Dec. 1986) 38-45.

[BBr] , P. Bak and R. Bruinsma, "One-dimensional Ising model and the complete devil's staircase,", Phys. Rev. Lett. 49 (1982) 249-251.

[Be] J. Bernoulli, "Sur une nouvelle espèce de calcul", Recueil pour les Astronomes 1 (Berlin) (1772) 255-284.

[BJa] P.M. Blecher and M.V. Jakobson, "Absolutely continuous invariant measures for some maps of the circle", in Statistical Physics and Dynamical Systems (Birkhäuser, Boston. Basel. Stuttgart, 1985).

[BlF] L. Block and J. Franke, "Existence of periodic points for maps of $\mathbf{S}^{1}$, " Inv. Math. $\mathbf{2 2}$ (1973) 69-73.

[BlGMY] L. Block, J. Guckenheimer, M. Misiurewicz, and L.S. Young, "Periodic points and topological entropy of one dimensional maps", in Springer Lecture Notes in Math. Vol. 819 (Springer, Berlin, 1980).

[Boh] P. Bohl, "Uber die hinsichtlich der unabhängigen variabeln periodische Differentialgleichung erster Ordnung", Acta Math. 40 (1916) 321-336.

[BBJ] T. Bohr, P. Bak, and M. H. Jensen, "Transition to Chaos by interaction of resonances in dissipative systems. II. Josephson junctions, charge-density waves, and standard maps." Phys. Rev. A 30 (1984) 1970-1981.

[Bow] R. Bowen, "Entropy for group endomorphisms and homogeneous spaces", Trans. Amer. 
Math. Soc. 153 (1971) 401-414.

[BFr] R. Bowen and J. Franks, "The periodic points of maps of the disk and the interval", Topology 15 (1976) 337-342 .

[Boyd] C. Boyd, "On the structure of the family of Cherry fields on the torus," Ergod. Th. \& Dynam. Sys. 5 (1985) 27-46.

[Boyl] P.L. Boyland, "Bifurcations of circle maps: Arnol'd tongues, bistability and rotation intervals," Commun. Math. Phys. 106 (1986) 353-381.

[BMT] K.M. Brucks, M Misiurewicz and C. Tresser, "Monotonicity properties of the family of trapezoidal maps," Commun. Math. Phys. 137 (1991) 1-12.

[BT] K.M. Brucks and C. Tresser, "A Farey tree organization of locking regions for simple circle maps," to appear in Prog. Amer. Math. Soc.

[BrB] R. Bruinsma and P. Bak., "Self-similarity and fractal dimension of the Devil's staircase in the one dimensional Ising model," Phys. Rev., B27 (1983) 5824-5825.

[CGT] A. Chenciner, J.M. Gambaudo and C. Tresser, "Une remarque sur la structure des endomorphismes de degré un du cercle," C.R. Acad. Sc. Paris t.299 série I (1984) $771-773$.

[Ch] E.B. Christoffel, "Observatio Arithmetica," Annali di Mathematica, 2nd series 6 (1875) 148-152.

[CTA] P. Coullet, C. Tresser, and A. Arnéodo, "Transition to turbulence for doubly periodic flows," Phys. Lett. 77A (1980) 327-331.

[DGMT] S. P. Dawson, R. Galeeva, J. Milnor and C. Tresser, "A monotonicity conjecture for real cubic maps," To appear in Proceedings of the NATO Advanced Study Institute on Real and Complex Dynamical Systems, Hillerød, June 1993. , IMS-SUNY Stonybrook preprint series, \# 1993/11 (1993).

[De] A. Denjoy, "Sur les courbes définies par les équations différentielles à la surface du tore," J. de Math. Pures et Appl. 11 (1932) 333-375.

[EKT] A. Epstein, L. Keen and C. Tresser, "The set of maps $F_{a, b}: x \mapsto x+a+\frac{b}{2 \pi} \sin (2 \pi x)$ with any given rotation interval is contractible," to appear in Comm. Math. Phys.

[FKS] M. J. Feigenbaum, L. P. Kadanoff, and S. J. Shenker, "Quasiperiodicity in Dissipative Systems: A Renormalization Group Analysis," Physica D 5 (1982) 370-386. 
[FT] B. Friedman and C. Tresser, "Comb structure in hairy boundaries: some transition problems for circle maps," Phys. Lett. A 117 (1986) 15-22.

[Ga] R. Galeeva, "Kneading sequences of piecewise linear bimodal maps," to appear in Chaos.

[Ga2] R. Galeeva, "House Maps' of the Interval," unpublished.

[GMT] R. Galeeva, M. Martens, and C. Tresser, "Inducing, slopes, and conjugacies," IMSSUNY Stonybrook preprint series, \# 1994/3 (1994).

[GaT] R. Galeeva and C. Tresser, "Piecewise linear discontinuous double coverings of the circle," Proc. Amer Math. Soc. 118 (1993) 285-291.

[GTr] J.M. Gambaudo and C. Tresser, "Transition vers le chaos pour les applications de degré un du cercle," in "le Chaos", Série Synthèses, (Editions Eyrolles, Paris, 1988).

[GB] L. Glass and J. Bélair, "Continuation of Arnold tongues in mathematical models of periodically forced biological oscillations," Nonlinear Oscillations in Biology and Chemistry, Lecture Notes in Biomathematics, edited by H. G. Othmer, (SpringerVerlag, Berlin, 1986) 232-243.

[Gl] L. Glass, "Cardiac arrhythmias and circle maps - A classical problem," Chaos 1 (1991) 13-19.

[GM] L. Glass and M. C. Mackey, From Clocks to Chaos, The Rhythms of Life, Princeton Univ. Press (Princeton, 1989).

[GoT] L. Goldberg and C. Tresser, "Rotation orbits and the Farey tree," Preprint I.B.M. (1991).

[HW] G.H. Hardy and E.M. Wright, "An Introduction to the Theory of Numbers," (Clarendon, Oxford, 1979).

[He1] M.R. Herman, "Mesure de Lebesgue et nombre de rotation," in Springer Lecture Notes in Math. Vol. 597 (Springer, Berlin, 1977). 271-293.

[He2] M.R. Herman, "Sur la conjugaison différentiable des difféomorphismes à des rotations," Pub. Math. I.H.E.S. 49 (1980) 5-234.

[He3] M.R. Herman, "Majoration du nombre de cycles périodiques pour certaines familles de difféomorphismes du cercle," An. Acad. Brasil. Ciênc. 57 (1985) 261-263.

$[\mathrm{HC}]$ D. Horton and D. K. Campbell, "Partial Period Doubling Sequences and Symbolic 
Dynamics in 'House Maps' of the Interval," to be published.

[Hu] C. Huyghens, letter to his father, dated February 26, 1665. Ouevres completes des Christian Huyghens, Vol. 5, p. 243 M. Nijhoff, ed.,(Societé Hollandaise des Sciences, The Hague, Netherlands (1893). (see references [Bak], [StSt] for a discussion of this observation).

[JBB1] M. Jensen, P. Bak, and T. Bohr, "Complete devil's staircase, fractal dimension and universality of mode-locking structure in the circle map," Phys. Rev. Letters 50 (1983) $1637-1639$.

[JBB2] M. Jensen, P. Bak, and T. Bohr, "Transition to chaos by interaction of resonances in dissipative systems. I. Circle maps," Phys. Rev. A 30 (1984) 1960-1969.

[Kh] K.M. Khanin, "Universal estimates for critical circle mappings," Chaos 1 (1991) 181186.

[MaT1] R.S. Mackay and C. Tresser, "Transition to topological chaos for circle maps," Physica 19D (1986) 206-237 \& 29D (1988) 427.

[MaT2] R.S. Mackay and C. Tresser, "Some flesh on the skeleton: the bifurcation structure of bimodal maps," Physica 27D (1987) 412- 422.

[MaT3] R.S. Mackay and C. Tresser, "Boundary of chaos for bimodal maps of the interval," J. London Math. Soc. 37 (1988) 164-181.

[Ma] A.A. Markoff, "Sur une question de Jean Bernoulli," Math. Ann. 19 (1882) 27-36.

[MTr] M. Martens and C. Tresser, "Forcing in $C^{0}([0,1])$ and renormalization in $P L([0,1])$," to appear.

[MSE] R. Mainieri, T. S. Sullivan, and R. E. Ecke, "Two-Parameter Study of the Quasiperiodic Route to Chaos in Convecting ${ }^{3} \mathrm{He}$ - Superfluid ${ }^{4} \mathrm{He}$ Mixtures", Phys. Rev. Lett. 63 (1989) 2357-2360.

[MT] M. Martens and C. Tresser, "Forcing of Periodic Orbits for Interval Maps and Renormalizations of Piecewise Affine Maps," SUNY StonyBrook, Institute for Mathematical Sciences, preprint \# 1994/17.

[MM] S. Martin and W. Martienssen, "Circle Maps and Mode Locking in the Driven Electrical Conductivity of Barium Sodium Niobate Crystals," Phys. Rev. Lett. 56 (1986) $1522-1525$ 
[MS] J. Masleko and H. L. Swinney, "A Complex Transition Sequence in the BelusovZhabotinskii Reaction," Phys. Scr. T9, (1985) 35-39.

[Me] W. de Melo, "Full families of circle maps," to appear.

[MSS] N. Metropolis, M.L. Stein, and P.R. Stein, "On finite limit sets for transformations on the unit interval," J. Comb. Theory 15 (1973) 25-44.

[MSt] W. de Melo and S. van Strien, One Dimensional Dynamics (Ergebnisse der Mathematik und ihrer Grenzgebiete. 3. Folge, Vol. 25) (Springer- Verlag, Berlin, 1993).

[MTh] J.Milnor and W. Thurston, "On iterated maps of the interval," in Springer Lecture Notes in Math. Vol. 1342 (Springer, Berlin, 1988).

[MH] M. Morse and G.A. Hedlund, "Symbolic dynamics II: Sturmian trajectories," Amer. J. Math. 62 (1940) 1-42.

[Mi1] M. Misiurewicz, "Horseshoes for mappings of the interval," Bull. Acad. Pol. Ser. Sci. Math. 27 (1979) 167-169.

[Mi2] M. Misiurewicz, "Twist sets for maps of the circle," Erg. Th. \& Dyn. Syst. 4 (1984) 391-404.

[MSz] M. Misiurewicz and W. Szlenk, "Entropy of piecewise monotone maps," Studia Math. 67 (1980) 45-68.

[MV] M. Misiurewicz and E. Visinescu, "Kneading sequences of skew tent maps," Ann.Inst. H.Poincaré 27 (1991) 125-140.

[Mu] P. Mumbrú, Estructura Periòdica i Entropia Topològica de les Aplicacions Bimodals Ph. D. Universitat Autònoma de Barcelona (1987).

[NPT] S. Newhouse, J. Palis and F. Takens, "Stable families of diffeomorphisms," Pub. Math. I.H.E.S. 57 (1983) 5-71.

[ORSS] S. Ostlund, D. Rand, J. Sethna, and E. D. Siggia, "Universal Properties of the Transition from Quasi-Periodicity to Chaos in Dissipative Systems," Physica 8D (1983) 303-342.

[PG] R. Perez and L. Glass, "Bistability, Period Doubling Bifurcations and Chaos in a Periodically Forced Oscillator," Phys. Lett. A 90 (1982) 441-443.

[Po] H. Poincaré, "Sur les courbes définies par des équations différentielles," J.Math.Pures et Appl. $4^{\text {eme }}$ série, 1 (1885) 167-244. Also in "Oeuvres Complètes, t.1" (Gauthier- 
Villars, Paris, 1951).

[STZ] R. Siegel, C. Tresser, and G. Zettler, "A decoding problem in dynamics and in number theory," Chaos 2 (1992) 473-493.

[Sm] H.J.S. Smith, "Note on continued fractions," The Messenger of Mathematics VI (1877) $1-14$.

[SHL] J.Stavans, F. Heslot, and A. Libchaber, "Fixed Winding Number and the Quasiperiodic Route to Chaos in a Convective Fluid," Phys. Rev. Lett. 55 (1985).x=

[St] S. H. Strogatz, "Norbert Wiener's Brain Waves," Lecture Notes in Biomathematics, Vol. 100, (Springer, 1994) 122-138.

[StSt] S. H. Strogatz and I. Stewart, "Coupled Oscillators and Biological Synchronization, Scientific American (1993) 102-109.

[Sw] G. Świạtek, "Rational rotation numbers for maps of the circle," Commun. Math. Phys. 119 (1988) 109-128.

[Tri] C. Tricot, "Douze définitions de la densité logarithmique," C.R. Acad. Sc. Paris t.293 série I (1981) 549-552.

[UC] D.J. Uherka and D.K. Campbell, "The sawtooth circle map," Preprint LA-UR 921491 (1992) (Unpublished).

[UTGC] D.J. Uherka, C. Tresser, R. Galeeva, and D.K. Campbell, "Solvable models for the quasi-periodic transition to chaos," Phys. Lett. A 170 (1992) 189-194.

[VdPVdM] B. Van der Pol and J. Van der Mark, "The heartbeat considered a a relaxation oscillation and an electrical model of the heart," Phil. Mag. 6, 763-775 (1968).

[Ve1] J.J.P. Veerman, "Symbolic dynamics and rotation numbers," Physica 134A (1986) $543-576$.

[Ve2] J.J.P. Veerman, "Symbolic dynamics of order-preserving orbits," Physica 29D (1987) 191-201.

[Ve3] J.J.P. Veerman, "Irrational rotation numbers," Nonlinearity 2 (1989) 419-428.

[VK] E.B. Vul and K.M. Khanin, "Homeomorphisms of the circle with singularities of break type," Russ. Math. Surveys 45 (1990) 229-230.

[Wi] A. T. Winfree, The Timing of Biological Clocks, (W. H. Freeman, New York (1987)).

$[\mathrm{YH}]$ W.-M. Yang and B.-L. Hao, "How the Arnold Tongues become sausages in a piecewise 
linear circle map," Commun. Theor. Physics (Beijing)8 (1986) 1-15. 


\section{FIGURE CAPTIONS}

Fig. 1: Typical tip and plateau maps: (a) tip or plateau map below the critical line,

(b) tip or plateau map on the critical line, (c) tip map above the critical line, (d) plateau map above the critical line.

Fig. 2: The 3-dimensional space $\mathcal{P}_{t}$ for the tip family parameters $(\Omega, S, \delta)$.

Fig. 3: The 3-dimensional space $\mathcal{P}_{p}$ for the plateau family parameters $(\Omega, U, \delta)$.

Fig. 4: Reinterpretation of certain critical maps.

Fig. 5: A non-monotonic left tongue boundary (of $A_{1 / 20}$, above the critical line, where $\delta=0.1$ for the tip family).

Fig. 6a: A portion of the $q^{t h}$ iterate, $f^{q}$, of a tip map, at the top of $L_{p / q}^{+}$.

Fig. 6b: A portion of the $q^{\text {th }}$ iterate, $f^{q}$ of a plateau map, at the top of $L_{p / q}^{+}$.

Fig. 7: The region of circular regularity for the tip family, for $\delta=1 / 4$. $(0 \leq p / q \leq 1 / 2$ with $q \leq 11$.)

Fig. 8: The surface $\Pi_{S^{q}}$ in $\mathbf{C}_{S^{q}}$. (The shaded triangle is a portion of $\Pi_{S^{q}}$.)

Fig. 9: The region of zero topological entropy for the tip family, for $\delta=1 / 4$. ( $0 \leq$ $p / q \leq 1 / 2$ with $q \leq 11$.)

Fig. 10: Numerically generated plateau $p / q$-stability regions, $A_{p / q}^{\oplus, s t a b}$, for $0 \leq p / q \leq$ $1 / 2, q \leq 5$.

Fig. 11: Construction of $f^{\prime}$ in the proof of Theorem 14, for the case $m=1$.

Fig. 12: Stable periodic orbits must attract a turning point.

Fig. 13: $A_{p / q}$ and $A_{p / q}^{s t a b}$ for $q \leq 5, \delta=1 / 4$.

Fig. 14a: $A_{p / q}^{s t a b}$ for $q \leq 11, \delta=1 / 2$. The portion of this figure above the critical line $S=S_{c}$ is $R_{1 / 2}$.

Fig. 14b: $A_{p / q}^{s t a b}$ for $q \leq 11, \delta=3 / 4$. The portion of this figure above the critical line $S=S_{c}$ is $R_{3 / 4}$. 
Fig. 15a: The neighbors of $A_{0 / 1}^{\text {stab }}$ unzipping from $A_{0 / 1}^{s t a b}$ at $\delta=\delta_{1}=1 / 2$.

Fig. 15b: The neighbors of $A_{1 / 2}^{\text {stab }}$ unzipping from $A_{1 / 2}^{\text {stab }}$ at $\delta=\delta_{2}=1-2^{-1 / 2}$.

Fig. 15c: The neighbors of $A_{1 / 3}^{s t a b}$ unzipping from $A_{1 / 3}^{s t a b}$ at $\delta=\delta_{3}=1-2^{-1 / 3}$.

Fig. 16: A circularly ordered chaotic 1/3-attractor and a coexisting stable 1/2-cycle for a tip map. $[(\Omega, S)=(0.4,2.2)$ and $\delta=1 / 2$.]

Fig. 17: The restriction of $t_{\Omega, S ; \delta}^{q(i)}$ to a $J_{k}$, in the proof of Theorem 18.

Fig. 18: The region $\mathbf{R}_{\delta}^{\prime}$ for $\delta=\delta_{3}^{\prime} \approx 0.1780$ is represented by the portion of this figure above the critical line $S=S_{c}$. It shows the unzipping of the extended 1/3-region from its neighbors. Its bigger neighbors unzip last.

Fig. 19: The structure of the parameter space for the tip maps around $L_{1 / 2}$ with $\delta>1 / 2$, as shown by the graphs of the second iterate of a lift of $t_{\Omega, S, \delta}$.

\section{... Appendix Figures:}

Fig. A1: Typical circle maps of degrees $-1,0,1$ and 2 along with their lifts.

Fig. A2: Monotone upper and lower bounds $F^{+}$and $F^{-}$for a lift $F$.

Fig. A3: Constructing a "stunted" family on the interval.

Fig. A4: Constructing a "stunted" family on the circle.

Fig. A5: Typical graphs of: $\alpha) C_{S, \mu ; a, b}$ and $\left.\beta\right)$ the corresponding $(a, b)$ parameter space.

Fig. A6: Successive approximations to the boundary of positive topological entropy in a stunted (also called cutting) family.

Fig. A7: Parameter space for the 3-dimensional stunted family $c_{S, \mu ; a, b}$ for fixed $S$.

Fig. C1: A 5 -cycle for $t_{\Omega, S ; 1 / 2}$ as $(\Omega, S)$ descends along the left boundary of $A_{2 / 5}$.

Fig. C2: A lift $F$ of $t_{\Omega, S ; \delta}$, where $\Omega=0$. 
Figures C3 to C15: Tip family stability regions $A_{p / q}^{s t a b}$ for $q \leq 7$ and for various $\delta$. ( $\delta=$ $.1, .2, .3, .4, .5, .6, .7, .8, .9, .95, .97, .99, .9999)$

Fig. C16: Two rational curves that intersect all $A_{p / q}$ below the critical line at nodes only. $(\delta=0.6$ and $q \leq 11)$

Fig. C17: Tip family regions $A_{p / q}^{s t a b}$ generated by the Monte Carlo algorithm, with $\delta=0.5$ and $q \leq 7$. (Compare with the exact regions shown in Figure C7.)

Fig. C18: Schematic view of plateau regions above the critical line.

Fig. C19: Boundary of topological chaos inside $A_{p / q}$ for the plateau family.

Fig. C20: Numerically generated sine family $p / q$-stability regions for $q \leq 5$. (Compare with Figure 10 for the plateau family.)

Fig. D1: Measure versus $S$ below the critical line, for $\delta=1 / 4,1 / 2,3 / 4$. 